Mitteilungen der Österreichischen Geographischen Gesellschaft, 162. Jg., S. 313-342

(Annals of the Austrian Geographical Society, Vol. 162, pp. 313-342)

Wien (Vienna) 2020, https://doi.org/10.1553/moegg162s313

\title{
Akteure und Perspektiven der Bananenwirtschaft auf La Palma
}

\author{
Norbert WeIXLBAUMER, Wien, und Anna WiPLINGER, Linz* \\ Erste Einreichung / initial submission: 01/2020; revidierte Fassung / revised submission: 11/2020; \\ endgültige Annahme / final acceptance: 12/2020 \\ mit $10 \mathrm{Abb}$. im Text
}

\section{INHALT}

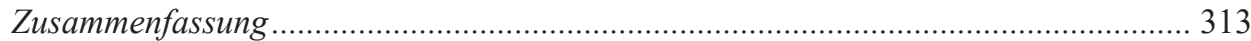

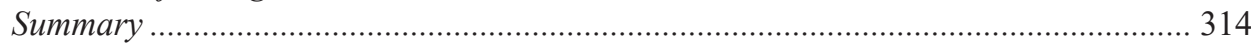

1 Einleitung .............................................................................................. 314

2 Rahmenbedingungen und Problemstellungen des Bananenanbaus auf La Palma ... 317

3 Bananenanbau auf La Palma ................................................................................. 320

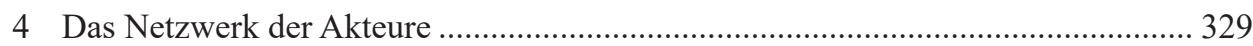

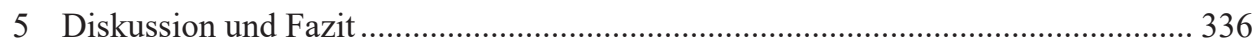

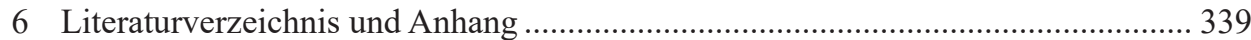

\section{Zusammenfassung}

Der Bananenanbau hat auf den westkanarischen Inseln, insbesondere auf La Palma, einen großen Einfluss auf Bevölkerung und Kulturlandschaft. Als Alternative bzw. in Ergänzung zur konventionellen Produktion etablierte sich seit Anfang der 2000er-Jahre die Bananen-Kultivierung gemäß den Kriterien des Biolandbaues. Ihr liegt ein spezifisches Akteursnetzwerk zugrunde, welches in dem vorliegenden Beitrag empirisch untersucht wird.

Mittels Befragungen verschiedener Persönlichkeiten aus den Bereichen Produktion, Vermarktung und Administration wird - flankierend zu explorativen Beobachtungen - im Rahmen von Experteninterviews erhoben, inwiefern die beteiligten Akteure und Akteursnetzwerke die Entwicklung der Bio-Banane fördern und welche Konflikte und Kontroversen dem Netzwerk in welcher Weise Grenzen setzen. Der Ansatz der Untersuchung und deren Ergebnisauswertung wurde in die Akteur-Netzwerk-Theorie und den „Follow the thing approach" eingebettet. Damit sollte - als zentraler Zielstellung des Artikels - der Tragfähigkeitsfrage bestehender und projektierter Netzwerke nachgegangen werden können.

\footnotetext{
* Ao. Univ.-Prof. Ing. Dr. Norbert Weixlbaumer, Universität Wien, Institut für Geographie und Regionalforschung, Universitätsstraße 7, A-1010 Wien; Mag. ${ }^{a}$ Anna WiPLINGER, Nietzschestraße 23, A-4020 Linz. E-Mail: norbert.weixlbaumer@univie.ac.at; anna.wiplinger@gmx.at.
} 
Auf Basis der Erhebungen konnte analysiert werden, wie sich die existenziellen Rahmenbedingungen des Netzwerks der Bio-Banane sowohl in der Nutzung diverser Ressourcen als auch in den Akteuren selbst sowie deren wechselseitigen Verknüpfungen widerspiegeln. Hinsichtlich der Zielsetzung der Akteure sind divergierende Motive und Absichten feststellbar. So stehen sich vor allem jene Akteure gegenüber, die sich aus umweltethischer Überzeugung für den ökologischen Anbau einsetzen, und solche, die sich vorrangig ökonomische Vorteile davon versprechen.

Schlagwörter: Bananenwirtschaft, Bananenanbau, Bio-Banane, Biolandbau, Akteursnetzwerke, Kanarische Inseln, La Palma

\section{Summary:}

\section{Actors and Prospects of the Banana Industry on La Palma}

On the Western Canaries, especially on the island of La Palma, banana production supports the livelihood of a significant proportion of the regional population and has a certain influence on the cultural landscape. Since the beginning of the $21^{\text {st }}$ century the organic banana was successfully introduced as a possible alternative and complement to the conventional cultivation. It is based on a specific actor-network, which will be the object of research of this article.

The empirical approaches applied included interviews with different people from the fields of production, marketing and administration as well as exploratory observations. This article aims at analysing the dynamics within the actor-network. It will be discussed how the individual actors support or impose limits to the network. The foundation of this research was determined through the "Actor-Network-Theory" as well as the "Follow the thing approach" which contribute to a better understanding of the complexity of the area of research. The prospects of the network's development will be analysed with regard to possibilities and potential obstacles.

The results of the investigation show that material and immaterial resources are as important as the actors who are connected by heterogeneous relations. The interlinking of all these components creates the main framework of the network of the organic banana. Concerning the actors' objectives there can be mainly identified two different intentions. Although there are numerous actors who support the organic cultivation of the banana for environmental and/or ethic reasons, we can also find as many actors who act particularly for economic reasons.

Keywords: Banana industry, banana cultivation, organic banana, organic farming, actor networks, Canary Islands, La Palma

\section{Einleitung}

Der Kanarische Archipel durchläuft seit Jahrzehnten einen Wandel, der durch einen gravierenden Verschleiß der Ressourcen Boden, Wasser und Luft sowie zahlreicher Küsten- 
zonen und der Arbeitskräfte gekennzeichnet ist - insbesondere aufgrund der bereits viele Jahrzehnte anhaltenden massentouristischen Entwicklung, die auf den einzelnen Inseln des Archipels allerdings sehr unterschiedlich ausgeprägt ist (vgl. RoDRíGUEZ_MARTín und Santamarta 2013; Dinerstein et al. 2017; HernándeZ Luis et al. 2017). Vor dem Hintergrund zahlreicher nicht-nachhaltiger Entwicklungen spielt die Landwirtschaft und hier der Bananenanbau - in seiner hauptsächlich praktizierten konventionellen Form mit all seinen bekannten Problemlagen (vgl. KoePPel 2008; Berners-Lee 2010; PiatTi FARnell 2016) - eine zentrale, die Ressource Kulturlandschaft beeinflussende Rolle. Der Biolandbau als eine relevante Nachhaltigkeitsstrategie bildet als Alternative dazu eine Option für eine tragfähigere Kulturlandschaftsentwicklung der Kanarischen Inseln.

Gemeinsam mit den neueren Entwicklungen in der Landwirtschaft können auch Maßnahmen der Raumplanung und hier insbesondere Schutzgebietsausweisungen und Schutzgebietsmanagement einiges zur zukunftstauglichen Entwicklung (vgl. HAMmER et al. 2018) des Archipels beitragen. So ist beispielsweise die gesamte Insel La Palma (Abb. 1), die von ihren Einwohnern wertschätzend ,Isla Bonita“ genannt wird, seit 2002 mit ihren rund 82.000 Einwohnern und 14 Gemeinden als Biosphärenreservat ausgewiesen. Die anderen zur Regionsverwaltung von Teneriffa gehörenden westlichen Inseln des Archipels wie El Hierro und La Gomera haben im Lauf des 21. Jahrhunderts ebenfalls auf Schutzgebietsausweisungen, gepaart mit naturnahem Tourismus und im Fall von El Hierro mit die gesamte Insel umfassenden erneuerbaren Energiegewinnungs-Initiativen, gesetzt. Im Gegensatz dazu positioniert sich Teneriffa - die wirtschaftlich bedeutendste Insel des Archipels - auch gerade hinsichtlich der Bananenwirtschaft als wenig zukunftstauglicher Hybrid zwischen „Schutz- und Schmutzraum“ (WeIXLBAumer 2005).

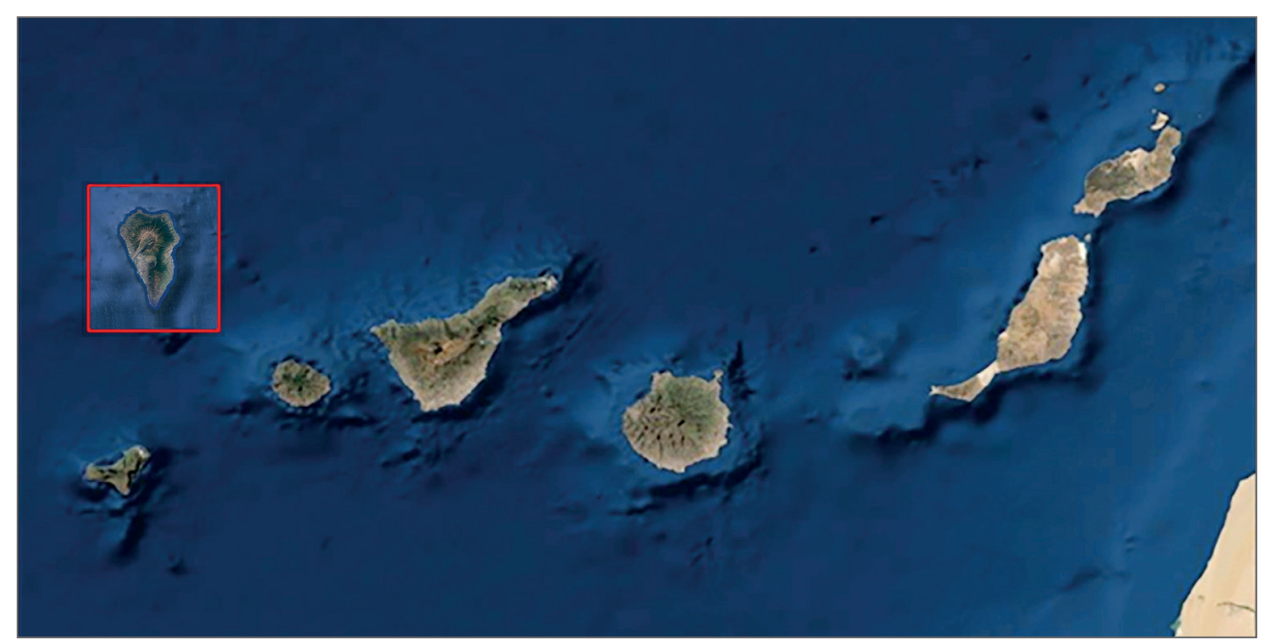

Quelle: Raúl Fernández de León (2020): Técnico GIS del Cabildo Insular de La Palma. http://www. cabildodelapalma.es

Abb. 1: La Palma, die nordwestlichste Insel der Inselgruppe der Kanaren; im Süden die Insel El Hierro, im Südosten die kleine Insel Gomera und östlich davon Teneriffa 
Vor dem Hintergrund der Biosphärenparkpolitik seit dem Jahr 2002 hat sich der Biolandbau auf La Palma - und generell auf den Kanarischen Inseln - stark dynamisiert (vgl. LóPEZ-CEPero 2006). Die Anzahl der Bioproduzenten allgemein hat sich in diesem Zeitraum nahezu verdreifacht. Auf La Palma, wo die Bananenpflanzungen hinsichtlich ihrer Flächenausdehnung und ihrer identitätsstiftenden Komponente die bedeutendste Rolle des gesamten Archipels einnehmen (vgl. WiPLINGER 2019), produzieren fünf Prozent aller Bananenbäuerinnen und -bauern mittlerweile ökologisch - mit steigender Tendenz. Der durch seine Terrassenkulturen und Kunststoffeinhausungen samt den spezifischen Infrastrukturen (z. B. Wasserzuleitungssysteme, Windschutzbauten) stark landschaftsprägende Bananenanbau ist aktuell die bedeutendste landwirtschaftliche Nutzungsform. Mehr als 6,6 Prozent der 28.893 Arbeitskräfte der Insel sind im Bananensektor tätig (ebd.).

Die seit Ende des 19. Jahrhunderts auf den Kanaren kultivierte Banane ist mittlerweile zum Marker von Kulturlandschaft und Küche geworden. Genauso gilt sie als Imageträger und Integrationsfaktor von La Palma als westlicher Peripherie des Archipels. Ihr besonderer Stellenwert spiegelt sich zudem nicht nur hinsichtlich der strengen phytosanitären Schutzmaßnahmen (damit über Importe keine Krankheiten eingeschleppt werden) wider, sondern darüber hinaus auch in der sprachlichen Zuordnung: Während nämlich die Kanarische Banane unter den Einheimischen als „plátano “ bekannt ist, fasst man die importierten Bananen unter dem Begriff „banana“ zusammen (vgl. RoBINSON und GALÁN SAÚCo 2011).

In diesem Beitrag geht es einerseits um die Rolle verschiedener Akteursgruppen im Transitionsprozess von konventioneller zu ökologischer Landbewirtschaftung am Beispiel des Bananenanbaus, andererseits um eine Analyse der fördernden wie auch begrenzenden Faktoren dieses Prozesses. Denn auch in absehbarer Zeit wird die konventionell produzierte Banane auf La Palma noch das Übergewicht bilden. Insgesamt werden folgende Fragen thematisiert: Wer sind - aus welchen Gründen bzw. mit welcher Motivation - die Akteure der Veränderung im Bananenanbau auf La Palma? Welche zentralen Akteursnetzwerke, die für die gegenwärtige Entwicklung verantwortlich sind, lassen sich herausarbeiten? Wie ist in der Biosphärenparkregion La Palma der Bio-Bananenanbau organisiert und welche Vernetzungen und Einstellungsmuster sind vorherrschend?

Die Methodik dieser Arbeit besteht zum einen aus Literaturrecherche und explorativen Beobachtungsreihen auf La Palma, zum anderen erfolgten im Frühjahr 2019 vor Ort 14 Tiefeninterviews. Diese dauerten zwischen 30 und 100 Minuten und wurden in der Landessprache mit Akteuren aus Produktion, Vermarktung und Administration durchgeführt (siehe Anhang). Die auf einen Tonträger aufgenommenen Interviews wurden nach den Transkriptionsregeln von DreHSING und PEHL (2018) verschriftlicht. Um die Auswertung der erhobenen Daten im Einklang mit den Grundsätzen der Akteur-Netzwerk-Theorie wie auch mit den Ansprüchen der explorativen Befragungsform von Experten zu gestalten, wurde die Qualitative Inhaltsanalyse nach MAYRING (2010) gewählt.

Als theoretisch-methodologischer Hintergrund dieses Beitrages dient der ,Follow the Thing Approach “ (vgl. Cook 2004; Colombino 2014; BenJAMIN und VIRKLER 2016). Es handelt sich dabei um eine Forschungsrichtung, die sich auf die Akteur-Netzwerk-Theorie (vgl. Callon 2006a, b; Latour 2010; Peuker 2011) bezieht und diese mit dem Ansatz verbindet, dass Objekte - in diesem Fall der Aktant „Kanarische Banane“ - über eine Art 
„Sozialleben“ und folglich eine historisch und geographisch-politisch relevante Biographie verfügen.

Im Folgenden wird nach einem Diskurs des Bananenanbaus und seiner regionsspezifischen Rahmenbedingungen auf die Akteure, deren Vernetzungen und Einstellungen zu einer auf stärkere Nachhaltigkeit ausgerichteten Bananenwirtschaft fokussiert. Dabei wird auf Ergebnisse der empirischen Erhebung Bezug genommen.

\section{Rahmenbedingungen und Problemstellungen des Bananenanbaus auf La Palma}

\subsection{Wasser als Basisressource des Bananenanbaus}

Das Temperaturregime von La Palma ist durch eine jährliche Durchschnittstemperatur von $16,3{ }^{\circ} \mathrm{C}$, mittlere Höchstwerte von $21,7^{\circ} \mathrm{C}$ im August und $13,7^{\circ} \mathrm{C}$ im Jänner gekennzeichnet. Grundsätzlich besteht jedoch eine starke Abhängigkeit der Temperatur von der jeweiligen topographischen Lage und Höhe. So können die Temperaturen im Norden und Süden der Insel um 15 Prozent von den genannten Werten abweichen (vgl. CIAP 2017, S. 64). Die Niederschlagsmenge ist vor allem von der Ausgestaltung des Reliefs wie auch der Exposition gegenüber den Passatwinden abhängig. Grundsätzlich sind die Niederschläge innerhalb von La Palma sehr unregelmäßig über das Jahr verteilt und erreichen ihren Höhepunkt in den Monaten Dezember und Jänner. Auf der Insel können zu dieser Zeit Spitzenwerte von durchschnittlich $200 \mathrm{~mm} / \mathrm{m}^{2}$ pro Monat gemessen werden (vgl. Marzol JaÉn und Máyer SuÁrez 2012, S. 407ff; AEMET 2012, S. 61-62). Da man in den Bananenpflanzungen - gemäß Aussagen der interviewten Produzenten - pro Staude täglich ca. 30 Liter Wasser benötigt, stellt dieses für die Bananenwirtschaft eine essenzielle Basisressource dar.

Die Wasserversorgung in La Palma speist sich vor allem aus der Entnahme von Grundwasser. Dieses wird laufend erneuert, weil durch die hervorragenden Infiltrationsbedingungen des pyroklastischen Bodenmaterials ein großer Teil des Regenwassers aufgenommen wird. Um das Grundwasser zu Tage zu fördern, bedient man sich entweder der Stollen (galerías) oder Brunnen (pozos). Die Stollen können mehrere Kilometer horizontal in das Vulkangestein hineinführen, fangen das Wasser der senkrecht im Gestein laufenden Wassergänge auf und transportieren es mithilfe einer leichten Abwärtsneigung hin zum Stollenausgang ab. Die Brunnen reichen bis zu mehreren hundert Metern in die Tiefe; gefördert wird das Wasser hier mithilfe von Turbinen und Pumpen (vgl. Rothe 2008, S. 15ff). Das Oberflächenwasser hingegen wird vor allem aus den Flussbecken der Kerbtäler gespeist und zu einem geringen Anteil in Staubecken, sogenannten balsas, gespeichert. Transportiert wird das Wasser über drei von öffentlicher Hand betriebene Hauptkanäle und private bzw. genossenschaftlich betriebene Leitungen.

Im Grunde verfügt La Palma über Wasservorkommen von ausgezeichneter Qualität für jeglichen Gebrauch (vgl. Gobierno de Canarias 2014). Bis vor wenigen Jahren galt La Palma als einzige Insel der Kanaren, deren Wasservorkommen bzw. -neubildung die Menge der Entnahmen noch überstieg, während sich die Grundwasservorkommen des Archipels 
insgesamt von $459 \mathrm{hm}^{3}$ im Jahr 1973 auf $290 \mathrm{hm}^{3}$ im Jahr 2012 verminderten (RoDRíGUEZ BRITO 2016, S. 250).

Auf La Palma wurden bis zum Jahr 2014 schätzungsweise $66,46 \mathrm{hm}^{3}$ Wasser pro Jahr aus den verschiedenen Quellen gefördert. Diese Menge wird auf etwa ein Drittel der gesamten Grundwasservorkommen geschätzt. 2014 beanspruchten der Bananenanbau und die übrige Landwirtschaft 85,4 Prozent der wertvollen Ressource, während man für die Industrie, die Tourismusbranche und den privaten Konsum 14,6 Prozent des jährlichen Verbrauchs verbuchte. Es werden jedoch nicht alle extrahierten Mengen auch tatsächlich genutzt. Eine nicht unbedeutende Quantität geht auf dem Transportweg wegen der mangelhaften Qualität der Leitungsinfrastruktur, aber auch durch unzureichende Speichermöglichkeiten verloren. Dies summierte sich auf durchschnittlich 7,43 $\mathrm{hm}^{3}$ pro Jahr (vgl. Gobierno de Canarias 2014, S. 227ff). La Palma ist innerhalb des Archipels die einzige Insel, die noch keine Entsalzungsanlage zur Süßwassergewinnung verwendet. Zudem gibt es momentan auf der „Isla Bonita“ auch keine Abwasseraufbereitung. Da jedoch seit dem Winter des Jahres 2008/09 die jährlichen Niederschläge vor allem im Winter immer wieder sehr spärlich ausfallen oder für mehrere Monate gar gänzlich ausbleiben, wurde die Wasserknappheit auch in La Palma zu einer viel diskutierten Problematik. Zudem ging der Grundwasserspiegel in den letzten Jahren kontinuierlich zurück, wie der Agraringenieur des „Consejo Insular de Aguas de La Palma“ Gómez Rodríguez im Interview konstatierte.

Die Wasserressourcen aus Oberflächenabfluss werden hauptsächlich von der Inselregierung zur Verfügung gestellt, während die Grundwasservorkommen von Genossenschaften und ein paar wenigen privaten Unternehmen gefördert, genutzt und verkauft werden (ebd.). Im Rahmen der Gesetzgebung ,Ley 12/1990, de 26 de julio, de Aguas “ soll eine öffentliche Verwaltung der Wasserversorgung die gerechte Verteilung der wichtigen Ressource garantieren. Dafür wurde für jede Insel ein Wasserrat mit eigener Rechtspersönlichkeit eingerichtet. Im Fall von La Palma ist das der Consejo Insular de Aguas de La Palma (CIAP). Zu seinen Aufgaben zählen das Management, die Planung und die Verwaltung der gesamten Wasservorkommen wie auch der Infrastruktur (vgl. BOE 1990, CIAP 2017).

Da die Rechte über den Zugang zum Grundwasser auf den Kanaren großteils über Beteiligungen an den jeweiligen Wassergemeinschaften organisiert sind, entstand im Lauf der Zeit ein Markt rund um die Ressource (vgl. Gobierno de Canarias 2014, S. 34-36). Die Preise auf dem Wassermarkt hängen grundsätzlich von Lage, Jahreszeit sowie Angebot und Nachfrage ab. So sind sie im Norden niedriger als im Süden der Insel, da dort der Großteil des Niederschlags fällt und somit auch der Transportweg kürzer ausfällt. Im Sommer steigen die Preise grundsätzlich aufgrund von Versorgungsengpässen und der steigenden Nachfrage. Der Preis für eine $p i p a^{1)}$ Wasser auf La Palma, die ohne Rechte am Markt zugekauft wird, kann den zu zahlenden Betrag derselben Menge Wasser, die im Rahmen der Mitgliedschaft bei einer Wassergenossenschaft erworben wird, um ein Vielfaches übersteigen.

\footnotetext{
1) Das Maß pipa wird exklusiv auf den Kanarischen Inseln hauptsächlich für die Angabe von Wassermengen verwendet. Eine pipa entspricht einer Kapazität von etwa 480 Litern.
} 


\subsection{Bedeutung und Kurzcharakteristik der Landwirtschaft}

Auf La Palma findet man vor allem an den Küstenabschnitten im Westen wie auch im Norden und Nordosten eine sehr gute Bodenqualität für landwirtschaftliche Zwecke. Es werden jedoch nur 50 Prozent der landwirtschaftlich nutzbaren Fläche tatsächlich landwirtschaftlich genutzt. Dabei nimmt der Bananenanbau mit 51 Prozent die meiste Fläche ein, gefolgt von Rebflächen, Obst und Gemüseanbau (vgl. Gobierno de Canarias 2018).

In der landwirtschaftlichen Produktion auf La Palma entfällt - wie auch auf den Kanaren insgesamt - der größte Anteil auf die Banane (vgl. Abb. 2). Auf der „Isla Bonita“ nimmt sie jedoch eine besonders dominante Stellung ein, da sie einen Anteil von 82,99 Prozent an der gewichtsbezogenen Gesamtproduktion hält, während dieser für den gesamten Archipel lediglich 47,72 Prozent beträgt.

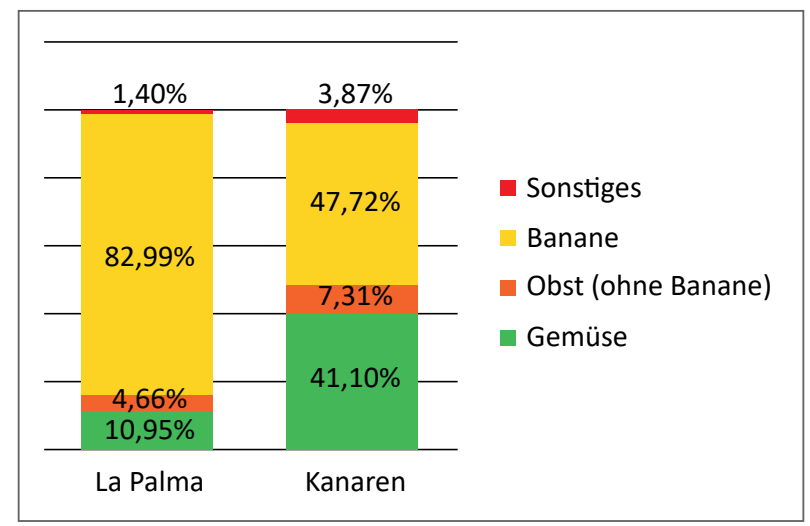

Quelle: Datengrundlage: ISTAC 2019; eigene Darstellung

Abb. 2: Gewichtsbezogene landwirtschaftliche Produktionsmenge nach Kategorien, La Palma und die Kanarischen Inseln insgesamt 2017

Der Anteil des Primären Sektors (Land- und Forstwirtschaft, Fischerei) am Bruttoinlandsprodukt unterscheidet sich in La Palma merklich von jenem der gesamten Kanarischen Inseln. Während er in Letzteren durchschnittlich 1,24 Prozent des BIP beträgt, fällt er auf La Palma mit 3,62 Prozent eindeutig höher aus. Die grundsätzliche Dominanz des Dienstleistungssektors lässt sich sowohl für La Palma als auch die Gesamtheit der Inseln feststellen, finden in diesem touristisch dominierten Sektor doch über 80 Prozent der Wertschöpfung statt.

Trotz der geringen Beteiligung des Primären Sektors am jeweiligen BIP muss festgehalten werden, dass dieser stark mit der Beschäftigung im Tertiären Sektor zusammenhängt. Denn der Dienstleistungsbereich beinhaltet viele Tätigkeiten, die eng mit der Vermarktung und dem Transport der produzierten Waren zusammenhängen. Somit ist ein Teil der Einkünfte im Tertiären Sektor untrennbar mit der Primärproduktion - allen voran dem Exportgut Banane - verbunden. Die Primärproduktion auf La Palma bietet außerdem 7,23 
Prozent der beschäftigten Bevölkerung einen Arbeitsplatz, während dies auf den Kanaren durchschnittlich lediglich 2,45 Prozent sind. In absoluten Zahlen stellte im Jahr 2016 die Landwirtschaft auf La Palma 2.088 von insgesamt 28.893 Arbeitsplätzen zur Verfügung (vgl. ISTAC 2019).

Bezüglich der ökologisch bewirtschafteten Fläche auf La Palma zeigt sich folgende Tendenz: Während im Jahr 1999 die ausgewiesene Fläche für Biolandbau nach AcostA (1999) bei 78 Hektar lag, konnte sie bis zum Jahr 2016 auf über 417 Hektar gesteigert werden (vgl. Gobierno de Canarias 2016). Damit beherbergte die „Isla Bonita“ zu dieser Zeit etwa 6,5 Prozent der Bio-Anbau- und Weideflächen des gesamten Archipels bzw. befanden sich 5,5 Prozent der genutzten landwirtschaftlichen Fläche auf der Insel im Ökolandbau. Die Anzahl der Bioproduzenten auf La Palma betrug 1999 erst 76 (vgl. ISTAC 2019) und im Jahr 2016 konnten bereits 257 Produzenten gezählt werden (vgl. CIAB 2019).

\section{Bananenanbau auf La Palma}

Die Banane wurde vermutlich im 15. Jahrhundert durch die Portugiesen von Guinea aus auf die Kanarischen Inseln gebracht (vgl. Vотн 1997; DoDo 2009). Im späten 19. Jahrhundert begann man dann auf dem Archipel mit dem intensiven Anbau. Eine stetig steigende Exportmenge konnte durch den monokulturellen Anbau, die Errichtung der nötigen Infraund Exportstruktur sowie durch fortlaufende technische Verbesserungen erreicht werden. Heute findet auf den Kanarischen Inseln, gefolgt von den französischen Überseeterritorien, Madeira und den Azoren, die größte Bananenproduktion innerhalb der EU statt.

\subsection{Charakteristika}

La Palma erfüllt die grundsätzlich erforderlichen Bedingungen für die Kultivation der Banane hinsichtlich des Klimas, der Wasservorkommen und der Böden. Da die Banane aber nur bis in Höhenlagen von etwa 400 Metern gut gedeiht, und die Kanarischen Inseln in vielen Zonen ihres ohnehin schon schroffen Reliefs eine große Hangneigung aufweisen, begann man bereits vor über hundert Jahren mit der Anlage von Terrassenparzellen. Somit konnte wertvolles Land zur landwirtschaftlichen Nutzung erschlossen werden. Heutzutage sind diese stufenartig angelegten Pflanzungen wichtiger Bestandteil der Kulturlandschaft von La Palma. Zudem bieten sie auch wertvollen Schutz vor Erosion und machen einen großflächigen Anbau erst möglich. Manche potenziellen Anbaugebiete, die bis auf die Bonität des Bodens alle relevanten Kriterien für den Bananenanbau erfüllen, werden mit fruchtbarem vulkanischem Material aus dem Inselinneren ergänzt. Diese künstlich geschaffenen Böden werden sorribas genannt.

Die Kanarische Banane wächst hauptsächlich auf kleinflächigen Parzellen, sogenannten minifundios. Für die Kanaren gilt, dass die Hälfte der schätzungsweise 8.000 Produzenten eine Parzelle von maximal einem Hektar bewirtschaftet (vgl. WIPLINGER 2019). Daneben ist auch das „Sharecropping“ eine beliebte Geschäftsform im Bananenanbau. Sie nennt sich contrato de aparceria und sieht vor, dass ein Bauer eine Pflanzung bewirt- 


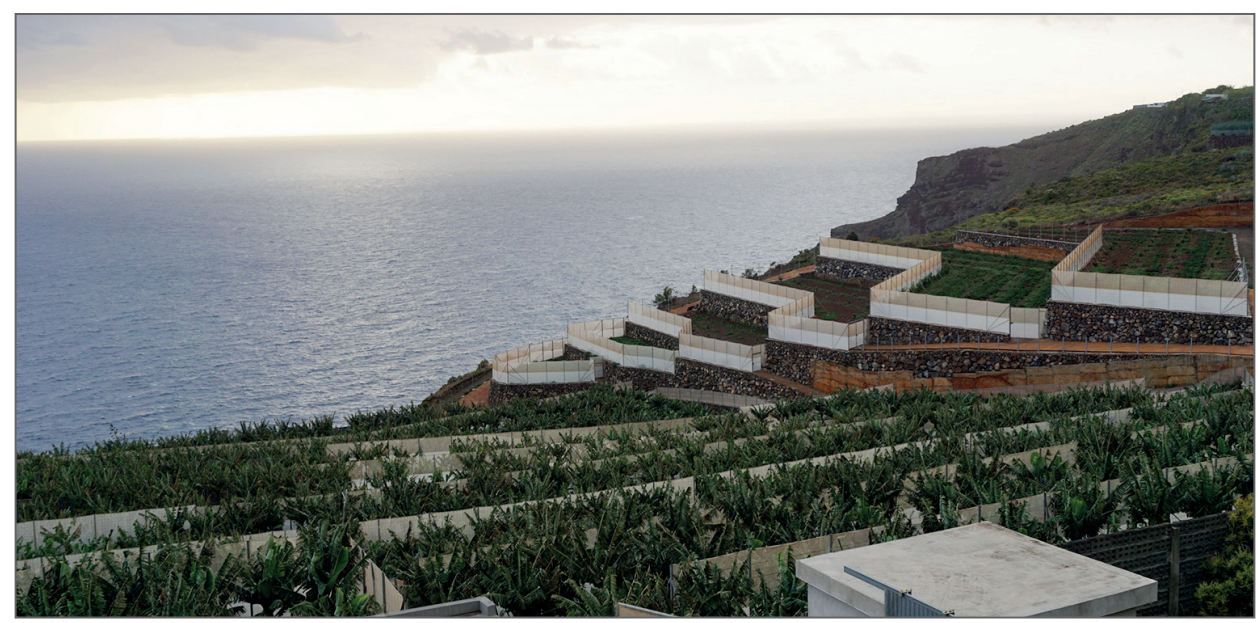

Quelle: Foto: WiPLINGER 2019

Abb. 3: Terrassenfeldbau und „Windschutz-Einhausungen“ von Bananenparzellen

schaftet, ohne diese zu besitzen. Er teilt sich mit dem Grundstückseigner sowohl Kosten als auch Einkünfte inklusive Subventionen und zahlt im Gegenzug keine Pacht (vgl. ebd.). Eine wichtige Schutzvorrichtung, die auf den Kanarischen Inseln oft zu finden ist, sind Gewächshäuser, sogenannte invernaderos. Ihre Errichtung ist zwar kostenintensiv, bietet aber Schutz vor extremen Wetterereignissen und Versalzung durch Meerluft. Weitere Vorteile sind eine geringere Evaporationsrate und eine frühere wie auch oft ertragsreichere Ernte durch die erhöhte Wärme innerhalb der weißen, luftdurchlässigen Einhausungen. Im Jahr 2017 befanden sich auf La Palma 722 von insgesamt 2.927 Hektar Bananenpflanzungen in einem Gewächshaus. Das entsprach rund 25 Prozent der gesamten Bananenanbaufläche (vgl. Gobierno de Canarias 2018, S. 25).

Da im Bereich der Dessertbananen weltweit der Trend zu Unterarten der Sorte Cavendish geht, finden sich diese auch in La Palma, großteils in Form der Pequeña Enana. Ihre Vorteile sind eine geringe Wuchshöhe, wodurch sie weniger Angriffsfläche für den Wind bietet und der Arbeitsaufwand bei der Pflege der Pflanze und auch der Ernte geringer ausfällt. Insgesamt wird dieser Unterart auch Robustheit gegenüber Krankheiten und Schädlingen nachgesagt. Weitere nennenswerte Sorten sind die Gran Enana oder auch die Gruesa (WIPLINGER 2019).

Grundsätzlich erfordert die Kultivierung von Bananen auf La Palma viel Handarbeit innerhalb der Pflanzungen, etwa beim Entfernen bzw. Versetzen von Sprösslingen, dem Entfernen der Infloreszenz wie auch der Blütenknospen jedes einzelnen Bananenfingers während der Reifung der Fruchtbüschel (piña). Manche Landwirte setzen auch auf das Einwickeln der Fruchtstände in Plastikhüllen, um Insekten abzuhalten oder das Wachstum voranzutreiben.

Bis heute kann unter anderem wegen der Hanglage bzw. Terrassierung keine maschinelle Bearbeitung zur Anwendung kommen. Die Versorgung mit Wasser findet hauptsäch- 
lich durch Druckbewässerung statt. Somit können im Gegensatz zur früher üblichen Staubewässerung - Überschwemmung des Bodens - große Mengen der kostbaren Ressource eingespart werden. Auf La Palma werden 100 Prozent der Bananenpflanzungen künstlich bewässert (vgl. Gobierno de Canarias 2018, S. 25). Als Vorteil der subtropisch und vergleichsweise isoliert gelegenen Kanaren gilt, dass schwerwiegende Krankheiten der Pflanzen bisher kaum vorkamen und so grundsätzlich weniger Pflanzenschutzmittel eingesetzt werden müssen (Vотн 1997, S. 52).

\subsection{Organisation der Produktion und Vermarktung}

Abbildung 4 bietet eine Übersicht über die verschiedenen Organisationsebenen der Produktion bis hin zu den einzelnen Schritten der Vermarktung von Bananen. Ebene 1 sind die Bauern. Sie gehören als Mitglied einer der Genossenschaften (Kooperativen) auch der nächsten Ebene - den Vertriebsgenossenschaften - an. An diese liefern und verkaufen sie nach Absprache regelmäßig ihre Bananen. Auf Ebene 2 befinden sich auch jene Vertriebsgenossenschaften, die für die Reinigung, die Verpackung und den Transport der Früchte zuständig sind. Neben dem operativen Geschäft übernehmen diese auch jegliche Kommunikation mit den Produzenten. Die Genossenschaften wiederum gehören in weiterer Folge einer O.P.P. (Organisation der Produzenten) der Ebene 3 an, einer übergreifenden Dachgenossenschaft. Sie trifft zukunftsweisende Entscheidungen, hat also das strategische Management inne. Dies impliziert die Kommunikation und Verhandlungen mit den Abnehmern, die Erschließung neuer Märkte sowie das Qualitätsmanagement. Die einzelnen O.P.P. können darüber hinaus Konzernen angehören. Zudem existiert noch der Dachverband ASPROCAN, der übergreifend für die O.P.P. und somit die gesamte Bananenproduktion der Kanarischen Inseln das Marketing, die Qualitätssicherung und das Management der Marke „Plátano de Canarias“ übernimmt (vgl. WiPLINGER 2019).

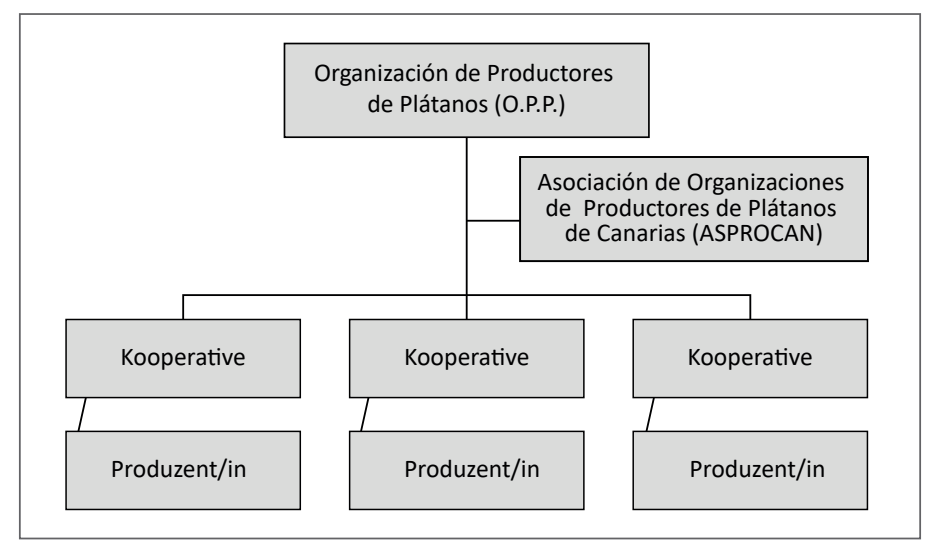

Quelle: eigene Darstellung

Abb. 4: Organigramm des Bananensektors auf den Kanarischen Inseln 
Auf La Palma operieren derzeit vier der insgesamt sechs kanarischen O.P.P., die mehr als 8.000 Genossenschaftsmitglieder zählen (vgl. ASPROCAN 2018). Dabei hält COPLACA (Grupo Regional de Cooperativas del Archipélago Canario) nicht nur auf La Palma den größten Marktanteil, sondern nimmt auch mit Abstand die Spitzenposition auf dem gesamten Archipel ein.

Bedeutend für die Vermarktung ist zudem die „Geschützte Geographische Angabe“ (g.g.A.). Die Verordnung Nr. 1151/2012 des Europäischen Parlaments und des Rates vom 21. November 2012 über Qualitätsregelungen für Agrarerzeugnisse und Lebensmittel hat sich zum Ziel gesetzt, EU-Erzeugnisse mit speziellen Charakteristika und in Hinblick auf ihre geographische Herkunft zu schützen (vgl. Europäisches Parlament und Rat der Europäischen Union 2012, S. 3ff.). Produkte, die unter die g.g.A. fallen, werden in Spanien mit dem Label in Abbildung 5 (linkes Bild) ausgezeichnet. Diese Qualitätsauszeichnung wurde den Bananen kanarischer Herkunft im Jahr 2013 verliehen. Sie tragen das Logo „Plátano de Canarias”, wie im rechten Bild von Abbildung 5 ersichtlich ist. Somit ist die kanarische Banane die einzige auf EU-Boden produzierte, der diese Qualitätsauszeichnung bisher verliehen wurde. Charakteristisch für die kanarische Banane sind sowohl der süße und aromatische Geschmack als auch die kleinen schwärzlichen Flecken auf der Schale (vgl. ASPROCAN o.J.).
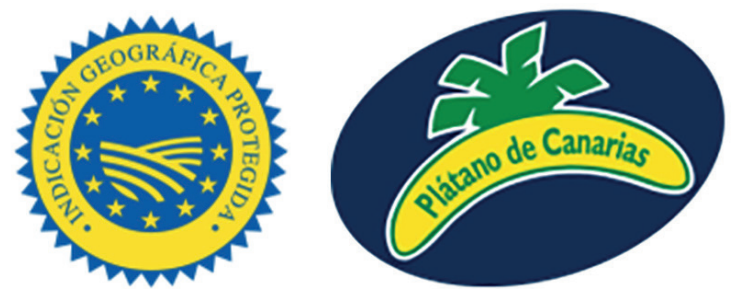

Quelle: linkes Bild: Europäische Kommission (o. J.); rechtes Bild: ASPROCAN (o. J.)

Abb. 5: linkes Bild: Label für Produkte aus EU-Erzeugung nach g.g.A. („Geschützte Geographische Angabe“); rechtes Bild: Logo der Herkunftsbezeichnung „Plátano de Canarias“" (Kanarische Banane)

\subsection{Anbauflächen und Produktion}

Auf La Palma nimmt die Banane mit 2.927 Hektar 4,1 Prozent der gesamten Inselfläche bzw. 51 Prozent der mit Feldfrüchten bestellten Fläche ein (vgl. Gobierno de Canarias 2018). Sie ist damit auf der „Isla Bonita“ die am häufigsten anzutreffende landwirtschaftliche Kultur. Die Banane auf La Palma nimmt auch in Relation zum gesamten Archipel - 1,2 Prozent der gesamten und 22,8 Prozent der gesamten aus Bananenpflanzungen bestehenden landwirtschaftlich genutzten Fläche der Kanaren (vgl. ISTAC 2019) - hinsichtlich ihrer Anbaufläche eine führende Rolle ein. Insgesamt wurden auf der „Isla Bonita“ 2017 etwa 34,4 Prozent der gesamten kanarischen Bananenmenge auf 32,3 Prozent der kanarischen Gesamtanbaufläche für Bananen produziert (vgl. ebd.). 


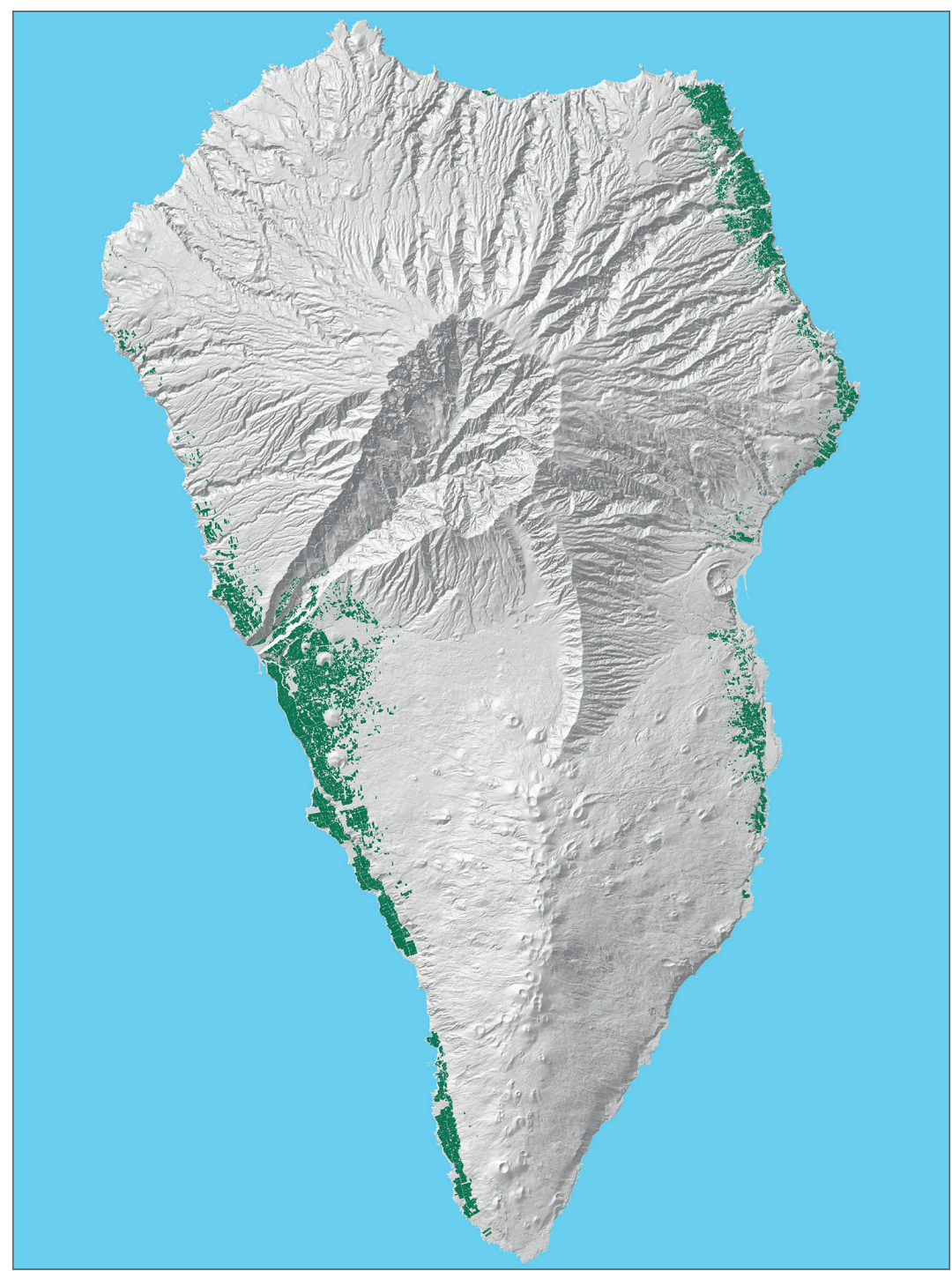

Quelle: Raúl Fernández de León (2020): Técnico GIS del Cabildo Insular de La Palma. - http:// www. cabildodelapalma.es

Abb. 6: Anbaugebiete der Banane („Plátano de Canarias“) auf der Insel La Palma, 2020

Wie in Abbildung 6 ersichtlich ist, befinden sich die Gebiete für die Bananenpflanzungen vor allem im niederschlagsreichen Nordosten und im Westen und Südwesten der Insel. Mit rund 38 Prozent wachsen mehr als ein Drittel der Bananenpflanzungen in Höhenlagen bis zu 100 Metern. Zwischen 100 und 300 Höhenmetern findet man etwa die Hälfte 
der Pflanzungen, während ab einer Höhe von 300 Metern mit rund 13 Prozent nur mehr vergleichsweise wenige Bananenpflanzungen vorhanden sind (vgl. Gobierno de Canarias 2018, S. 18f).

\subsection{Sozioökonomische Bedeutung}

Hinsichtlich der Arbeitsmarktentwicklung im Bananenanbau von La Palma bzw. auf den Kanarischen Inseln wurden noch keine konkreten Zahlen veröffentlicht. César Martín, der Direktor der landwirtschaftlichen Abteilung der kanarischen Regierung, spricht im Interview von mehr als 8.000 Produzenten auf den Kanaren und der doppelten Anzahl an indirekt damit verbundenen Arbeitsplätzen. Im Fall von La Palma schlagen RodríGUEZ GoNZÁLEZ et al. (2016) eine Berechnung der nötigen Arbeitsstunden in Kombination mit dem Flächenverbrauch der Bananenkultur zur Evaluierung potenzieller Arbeitsplätze vor. Im Durchschnitt verlangt eine konventionell bewirtschaftete Fläche von einem Hektar einen Arbeitskräfteeinsatz von 1.127 Stunden. Als Vollzeitbeschäftigung wird ein Wert von 1.764 Arbeitsstunden herangezogen (vgl. ebd.). Nach dieser Berechnungsmethode ergäbe sich für die Bananenproduktion auf La Palma ein Mindestbedarf von 1.871 und für die Kanaren von 5.798 Arbeitskräften.

Somit müssten gemäß dieser Berechnungsmethode 91 Prozent der 2.088 Arbeitskräfte in der Landwirtschaft La Palmas im Bananensektor arbeiten. Für den gesamten Arbeitsmarkt der Insel würde das bedeuten, dass mehr als 6,6 Prozent der 28.893 Arbeitskräfte in der Bananenproduktion beschäftigt sind. Für die Kanarischen Inseln lässt sich ermitteln, dass zumindest 29,6 Prozent der 19.500 Beschäftigten des Primären Sektors bzw. 7,3 Prozent der gesamten 795.000 Arbeitsplätze in der Bananenproduktion zu finden sind (Stand 2016, vgl. ISTAC 2019). Noch nicht inbegriffen sind in all diesen Berechnungen indirekte Dienstleistungen wie etwa der Handel mit Dünge- und Spritzmitteln, Verpackung, Transport etc. Diese Tätigkeiten schaffen schätzungsweise die doppelte Anzahl der Arbeitsplätze wie sie in der Produktion gebraucht werden.

\subsection{Marktsituation}

Vor allem in der zweiten Hälfte des 20. Jahrhunderts konnte die überseeische „Dollar-Banane“ große Anteile am europäischen Bananenmarkt einnehmen. Die Produzenten aus Übersee schafften es vor allem durch weiterentwickelte Transporttechniken und niedrigere Stückkosten, viele europäische Abnehmer für sich zu gewinnen. Die kanarische Bananenproduktion hingegen, im Zuge des Zweiten Weltkrieges auf den spanischen Binnenmarkt konzentriert, zeichnete sich zu dieser Zeit durch eine schlechte Organisation, viele Kleinbetriebe und kostenintensive Produktionsbedingungen aus (vgl. Vотн 1997, S. 210ff; Dodo 2009, S. 17f).

Mit dem Beitritt Spaniens zur EU im Jahr 1986 fielen darüber hinaus die Import-Beschränkungen für ausländische Bananenimporte in das spanische Inland weg. Um jedoch europäische Hersteller zu schützen, wurde 1993 die erste EU-Bananenverordnung 
ins Leben gerufen. Diese umfasste Maßnahmen, wie etwa die Etablierung gemeinsamer Qualitätsnormen, finanzielle Förderungen für die Bäuerinnen und Bauern, eine Verbesserung der Organisationsstruktur europäischer Produzenten und Händler sowie auch Importbeschränkungen für ausländische Exporteure (vgl. VoтH 1997, S. 216ff). Nach mehreren Adaptierungen gilt nunmehr eine Zollgebühr für den Import von Bananen aus Drittländern, während die AKP-Länder ${ }^{2)}$ noch von einer zoll- und kontingentfreien Einfuhr ihrer Früchte in die EU profitieren (vgl. Europäische Kommission 2010 und 2019).

Um mit den niedrigen Preisen und höheren Produktionsmengen der Konkurrenz mithalten zu können, erhalten die Bäuerinnen und Bauern bereits seit der Etablierung der Bananenverordnung von 1993 finanzielle Rückendeckung seitens der Union im Rahmen des „POSEI-Programms“ (zur Lösung spezifischer auf die Insellage zurückzuführender Probleme). Die „Verordnung (EU) Nr. 228/2013 des europäischen Parlaments und des Rates vom 13. März 2013 über Sondermaßnahmen im Bereich der Landwirtschaft zugunsten der Regionen in äußerster Randlage der Union und zur Aufhebung der Verordnung (EG) Nr. 247/2006 des Rates“ ermöglicht Maßnahmen zur Förderung der Landwirtschaft, und zwar in Gebieten mit geringer Fläche, schwierigen orographischen und klimatischen Bedingungen und einer wirtschaftlichen Abhängigkeit von wenigen Erzeugnissen.

$\mathrm{Zu}$ diesen Regionen in äußerster Randlage gehören auch die Kanarischen Inseln. Die Regionalregierung spricht dem Bananensektor eine jährliche finanzielle Förderung von 114,1 Millionen Euro zu, die je nach Produktionsmenge direkt an die Landwirte ausbezahlt wird. Darin inkludiert ist bereits eine zusätzliche Unterstützung für den Ertrags-eingeschränkten Freiluft-Anbau, also für Pflanzungen außerhalb der Gewächshäuser. Die Summe von 1.200 Euro pro Hektar wird jährlich ausbezahlt und es gilt eine Gesamtgrenze von 7.600 Hektar (vgl. Gobierno de Canarias 2015, S. 38f). Für die Kanaren hätte das bedeutet, dass im Jahr 2017 bei einer Produktion von 421.297 Tonnen ${ }^{3)}$ mit dem Abzug der Förderung von Freiluftflächen eine Subvention von 0,25 Euro pro Kilogramm Banane zur Verfügung stand. Dies bedeutete für La Palma im Jahr 2017 eine Förderung des Bananensektors in der Gesamthöhe von 39,5 Millionen Euro.

Im Zeitraum 2010 bis 2018 nahmen die Bananeneinfuhren in die EU kontinuierlich zu. 2018 kamen die Importe von über 6.548.643 Tonnen Bananen in die EU zu 75 Prozent aus dem lateinamerikanischen Nicht-EU-Raum, während die AKP-Länder mit etwa 16 Prozent und die EU-Produzenten mit lediglich 9 Prozent vertreten waren. Bezüglich des Marktwertes erreichten die EU-Bananen hingegen einen Anteil von 11,3 Prozent, während jene der AKP-Länder 17,6 Prozent und der Drittländer 71,1 Prozent des Marktvolumens einnahmen. Unter den EU-Produzenten nimmt die kanarische Banane eine Schlüsselrolle ein, trägt sie doch mit 65,3 Prozent zur gesamten Produktion im EURaum bei (vgl. Europäische Kommission 2019).

2) Die AKP-Ländergruppe (AKP: Organisation Afrikanischer, Karibischer und Pazifischer Staaten) umfasst zurzeit 79 Länder in Afrika, der Karibik und dem Pazifik - zumeist frühere Kolonien Frankreichs und Großbritanniens -, mit denen die EU (früher EG) im Zuge ihrer gemeinsamen Handelspolitik ab 1975 stark entwicklungspolitisch motivierte Handelsabkommen geschlossen hat.

3) Da in der Verordnung ein Grenzwert von 420 Millionen Kilo jährlich festgelegt ist, konnte die Subvention nur für 99,69 Prozent der Produktion berechnet werden. 
Das spanische Festland ist der größte Abnehmer für die kanarischen Bananen. Im Jahr 2017 importierte es 87,3 Prozent der auf den Kanaren produzierten Bananen, nur 8,6 Prozent wurden auf den Inseln selbst konsumiert und lediglich 0,3 Prozent wurden außerhalb Spaniens vermarktet. Im Hinblick auf La Palma orderte das spanische Festland 95 Prozent der produzierten Früchte. Der lokale Markt nahm schwache 0,5 Prozent der Bananen auf, das Ausland lediglich 0,25 Prozent.

\subsection{Wertschöpfung}

Die Wertschöpfungskette im Bananensektor besteht aus fünf Stufen (vgl. Abb. 7). Die Produzenten verkaufen an die heimischen Genossenschaften bzw. O.P.P.s, welche wiederum an Großhändler im Zielland liefern. Diese kümmern sich nicht nur um die Reifung der Früchte, sondern vertreiben die Ware an die einzelnen Supermarktketten bzw. Lebensmittelläden. Die Einzelhändler bieten die Ware den Endkonsumenten an.

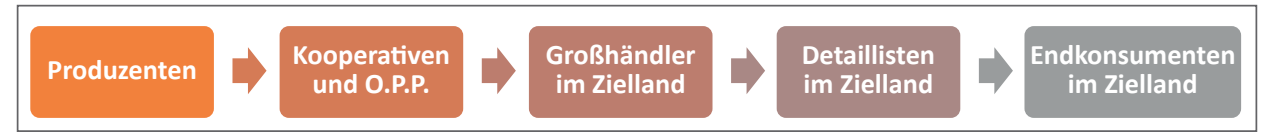

Quelle: eigene Darstellung

Abb. 7: Die Wertschöpfungskette in der Bananenvermarktung

Die Preise für kanarische Bananen können sowohl von Jahr zu Jahr als auch monatlich stark schwanken. Wie Abbildung 8 zeigt, konnten die Bäuerinnen und Bauern im Jahresdurchschnitt von 2005 bis 2018 minimal 0,33 und maximal 0,61 Euro pro Kilogramm mit ihren Früchten erzielen, die Großhändler zwischen 0,88 und 1,34 Euro, während der Betrag im Einzelhandel zwischen 1,70 und 2,40 Euro schwankte.

Vom Endverkaufspreis entfallen also etwa 45 Prozent auf den Einzelhandel, 31 Prozent auf den Großhandel und rund 24 Prozent auf die Produzenten. Die jeweiligen Anteile an der Wertschöpfungskette sagen jedoch nichts über die Gewinnspanne aus, denn dazu müssten noch die jeweiligen Kosten abgezogen werden. Diese setzen sich bei den Großhändlern aus Reifung, Lagerung, Verpackung und Transport zusammen. Die Detaillisten vor Ort wiederum zählen vor allem Arbeitskräfte in den einzelnen Filialen zu ihren kostenverursachenden Faktoren (vgl. Ministerio de Agricultura, Pesca y Alimentación 2012).

Im Jahr 2017 wurden durch die kanarischen Bananenbauern insgesamt Einkünfte von 291 Millionen Euro generiert. Auf La Palma waren dies 100,5 Millionen Euro, die zu 60,6 Prozent aus den regulären Einkünften und zu 39,4 Prozent aus den Subventionen bestanden. Während auf den Kanaren die jährlichen regulären Einkünfte pro Hektar 19.499,98 Euro exklusive Subventionen betrugen, waren dies auf La Palma 20.821 Euro pro Hektar (vgl. Ministerio de Agricultura, Pesca y Alimentación 2019). Die Wertschöpfung der Bananenproduktion pro Hektar auf La Palma übersteigt somit um 6,78 Prozent jene des gesamten kanarischen Bananensektors. 


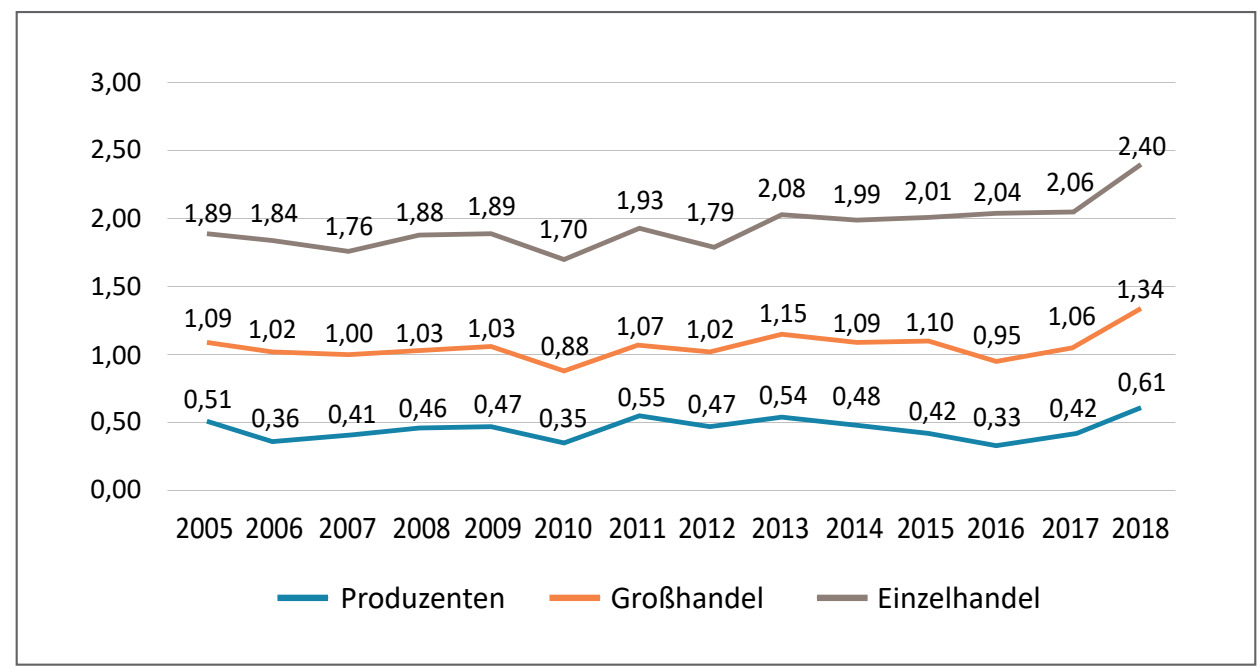

Quelle: Datengrundlage: Ministerio de Agricultura, Pesca y Alimentación (2019); eigene Darstellung

Abb. 8: Preisentwicklung der Kanarischen Banane im Zeitraum von 2005 bis 2018 (in Euro pro Kilogramm)

\subsection{Nicht-nachhaltige Bananenproduktion}

Die konventionelle Kultivierung von Bananen wird oftmals angegriffen, weil sie input-intensiv ist, in Monokultur betrieben wird und vor allem einen hohen Dünge- und Pestizideinsatz erfordert (vgl. KoEPPEL 2008). Sie steht in der Kritik, alles andere als nachhaltig zu sein, da sie überdies auch einen hohen Infrastruktureinsatz (z. B. Einhausungen aus Kunststoff) und Wasserverbrauch aufweist. Der durchschnittliche Bedarf auf den Pflanzungen beträgt 1.260 Liter $/ \mathrm{m}^{2}$ pro Jahr (vgl. LóPEZ-CEPERO 2006 et al.).

Neben negativen Auswirkungen auf die Umwelt, die Biodiversität und die menschliche Gesundheit können darüber hinaus besonders in Zeiten der Überproduktion die Kosten für all die eingesetzten Mittel nicht durch den Verkaufspreis gedeckt werden. Die jährlichen Ausgaben allein für Pflanzenschutzmittel betragen pro Produzent im Jahr zwischen 1.500 und 2.000 Euro (Auskunft von Interviewpartner Batista Rodríguez - Agraringenieur). Einen finanziellen Ausgleich bieten im Moment die Subventionen der EU im Rahmen des „POSEI-Programms“, allerdings besteht hier die Gefahr einer langfristigen Förder-Abhängigkeit. Die Subventionen des Bananensektors spielen eine bedeutende Rolle in der Wirtschaft der Kanaren und besonders auf La Palma, da ein großer Teil der Arbeitsplätze direkt oder indirekt vom Anbau der gelben Frucht abhängt.

Als alternatives Modell zum umstrittenen konventionellen Anbau konnte sich seit Anfang der 2000er Jahre die in den folgenden Ausführungen zu diskutierende und auf die Akteursstrukturen La Palmas konkret Bezug nehmende Bio-Bananenproduktion etablieren. 


\section{Das Netzwerk der Akteure}

\subsection{Bio-Bananenanbau im Spiegel der Akteure ${ }^{4)}$}

Die Produktion von Bio-Bananen bedarf einer umfangreichen Handarbeit und geht sowohl in freien und terrassierten Pflanzungen als auch in Gewächshäusern vonstatten. Die Bio-Banane, welche auch zur Marke „Plátano de Canarias“ gezählt wird, durchläuft innerhalb der Vermarktungskette dieselben Stationen wie die konventionelle Frucht, unterscheidet sich jedoch hinsichtlich anderer - insbesondere produktionsbezogener Rahmenbedingungen von ihr. Da es auch in der Bananenproduktion viele verschiedene Möglichkeiten zur Realisierung des Ökolandbaus gibt, können an dieser Stelle nicht alle Varianten in ihrer Gesamtheit ausgeführt werden, denn jede Bäuerin und jeder Bauer wirtschaftet anders, und somit sind auch die zugehörigen Bananenfincas sehr individuell gestaltete Agrarökosysteme (Interview mit Batista Rodríguez).

\section{„Es besteht [im Biolandbau] eine große Diversität hinsichtlich der Einstellung wie auch der praktischen Herangehensweise, somit lassen sich keine Generalisierun- gen ableiten. “ (Francisco García Lázaro, Bio-Bauer) ${ }^{5)}$}

Hinsichtlich des Wasserkonsums berichten sowohl die Bio-Bäuerin Martina Rasi-Rüger als auch der Bio-Bauer Francisco García Lázaro, dass mit einer geeigneten Bodenbedeckung durch Mulchen oder Vegetation der Verbrauch insgesamt gesenkt werden kann. Die Wurzeln der Begleitpflanzen fördern die Wasserspeicherung im Boden und halten die Evaporationsrate gering. Viele der benötigten Nährstoffe lassen sich bereits auf der eigenen Finca finden, nämlich in Form von diversen Pflanzenresten oder als Dung aus der Tierhaltung. Diese Form der Wiederverwendung entspricht zumindest teils dem Kreislaufgedanken der biologischen Landwirtschaft (Interview mit LorenzoRodríguez). Ist von beidem nicht genug innerhalb der eigenen Pflanzung vorhanden, so können der Dünger bzw. einzelne Komponenten auch zugekauft werden (Interview mit Pérez Díaz). Auf La Palma gibt es die Möglichkeit, sich tierische Abfälle aus den umliegenden Tierhaltungsbetrieben zu beschaffen oder die einzelnen Nährstoffe bei Unternehmen oder den eigenen Genossenschaften als Biodünger zuzukaufen (Interview mit Rasi-Rüger).

Insgesamt fallen die Kosten für jegliche Inputs im ökologischen Anbau der Banane auf La Palma im Vergleich mit einer konventionellen Pflanzung geringer aus, eine gute Handhabung der Finca vorausgesetzt (Interview mit López-Cepero Jiménez). Bedient man sich jedoch externer betrieblicher Produktionsmittel, die für den biologischen An-

4) Die in den Kapiteln 4.1 und 4.2 in Kursivschrift angeführte Zitate beziehen sich auf die Aussagen von Interviewpartnern im Rahmen der 2019 durchgeführten Tiefeninterviews vor Ort. Eine Liste der Interviewpartner und ihrer Funktion findet sich im Anhang zu diesem Beitrag. Die Übersetzung des spanischen Originaltexts erfolgte durch die Autoren.

5) Spanischer Originaltext: ,Entonces hay mucha diversidad de sentimientos, de formas de trabajar y de forma de hacerlo. Con lo cual no se puede generalizar. " 
bau zugelassen sind, so können die Kosten dafür sogar jene des konventionellen Anbaus übersteigen (vgl. LóPEZ-CEPERO JIMÉNEZ et al. 2006).

Im Ökolandbau wird allgemein auf systemisch wirkende synthetische Pflanzenschutzmittel verzichtet. Alle interviewten Bio-Bananenproduzenten bauen stattdessen auf der eigenen Finca selektiv Pflanzen zur Insektenabwehr an oder kaufen maximal etwas Schwefel zum Auftragen auf die Stauden zu. Neben diesen altbewährten Mitteln oder dem Waschen der Pflanzen mittels Druckbewässerung ist beim Ökolandbau besonders die Forschung essenziell. Denn immer wieder tauchen auch neue Plagen wie aktuell der picudo, eine Unterart des Rüsselkäfers, auf, gegen die erst wirksame Abwehrstrategien entwickelt werden müssen (Interview mit Batista Rodríguez). Somit lässt sich schließen, dass neben den materiellen Voraussetzungen auch „traditionelles Wissen“ und das jeweilig erforderliche spezifische Know-How als immaterielle Ressource eine grundlegende Rolle im Anbau der Bio-Banane von La Palma einnehmen. Bestätigt wird dies von Agraringenieur Lorenzo Rodríguez, der vor allem das Bewusstsein und das Wissen um einen tragfähigen Anbau hervorhebt:

\begin{abstract}
„,...] Die Bewirtschaftung einer biologischen Finca erfordert tatsächlich ein höheres Maß an Arbeitseinsatz als bei einer konventionellen. Dessen sollte man sich bewusst sein und es sollte so kommuniziert werden. Um biologische Landwirtschaft erfolgreich zu betreiben, muss man einfach ein guter Bauer sein. Steigt man also auf den biologischen Anbau um, sollte man ein Mindestmaß an Fachkenntnissen und auch das nötige Bewusstsein dafür mitbringen. " (Lorenzo Rodríguez) $)^{6}$
\end{abstract}

Entgegen mancher Vorurteile verlangt der biologische Anbau der Banane auf der „Isla Bonita“" insgesamt einen höheren Arbeitseinsatz, der mit einer ausreichenden Wissensbasis einhergeht. Dies gilt vor allem für das erste Jahr der Umstellung (Interview mit Méndez García). Auch Brito Pérez zufolge sollte sich jeder Landwirt darüber im Klaren sein, dass im Ökolandbau bestimmtes Know-how und Kompetenzen die Voraussetzung für eine erfolgreiche und langfristige Bewirtschaftung sind (Interview mit Brito Pérez). Zudem gehören zu biologischer Landwirtschaft sicherlich eine gewisse Portion Experimentierfreudigkeit und Offenheit gegenüber neuen Methoden und Ideen (Interview mit Rasi-Rüger). Insbesondere gilt dies für die Kultivierung der Banane, da diese keine einfach zu handhabende Kulturpflanze ist. Zudem ist der Bio-Bananenanbau kein abgeschlossenes landwirtschaftliches Konzept, sondern eine fortlaufende Entwicklung auf Basis von „Trial and Error". Somit stellen auch „Good-Practice“-Beispiele eine wichtige Grundlage für den vergleichsweise noch jungen Zweig der biologischen Landwirtschaft auf der Insel La Palma dar (Interview mit Batista Rodríguez).

Verlässt die Banane das Feld, so wird sie in einer der Genossenschaften für den weiteren Transportweg vorbereitet. Jede von ihnen, die eine biologische Linie in ihrem Sortiment platziert hat, muss über ein eigenes Förderband zur Verarbeitung der Früchte verfügen.

\footnotetext{
6) Spanischer Originaltext: „,...] el manejo de una finca en ecológico en realidad lleva más trabajo que en convencional. Yo creo que eso hay que tenerlo claro y hay que decirlo así. Y hay que ser buenos agricultores para saber hacer agricultura ecológica. Se puede uno pues, pasar pero hay que tener conocimientos y después conciencia de lo que se va a hacer. "
} 
Denn die Bio-Banane wird neben Wasser lediglich mit natürlichen Zusätzen gewaschen und darf nicht mit den Rückständen der konventionellen Ernte in Berührung kommen. Genauso müssen alle Schutzvorrichtungen in den Bananenkisten aus umweltfreundlichen bzw. kompostierbaren Materialien bestehen. Als zentrale Inputs der Packstellen gelten vor allem Wasser, Energie, Pflanzenschutzmittel, Plastik und Karton (Interview mit Arenciaba Sánchez).

\subsection{Akteure und Zukunftsperspektiven}

Abbildung 9 gibt eine Übersicht über die Hauptakteure, die an der Bildung von Netzwerken und der Erweiterung des Anbaus der Bio-Banane von La Palma beteiligt sind. Miteinbezogen wurden all jene Akteure, denen im Zuge der explorativen Beobachtung und der Experteninterviews Handlungsfähigkeit und Wirkmacht postuliert wurde. Denn als Akteur gilt der Akteurs-Netzwerk-Theorie zufolge schließlich nicht nur eine Entität, „die von vielen anderen zum Handeln gebracht wird“ (LATOUR 2010, S. 81), sondern auch selbst Handlungsfähigkeit besitzt. Ein Akteur ist imstande, weitere Bestandteile des Netzwerks in Verbindung zueinander zu setzen und diese Verknüpfungen zu transformieren (vgl. Callon 2006b, S. 318).

Dem Anspruch der sogenannten „Generalisierten Symmetrie“ folgend, werden sowohl menschliche als auch nicht-menschliche und hybride Akteure berücksichtigt. Jeder von ihnen kann in weiterer Folge auch ein ,punktualisiertes“ Akteurs-Netzwerk darstellen. Da insbesondere die Produzenten eng mit der Bio-Banane selbst zusammenhängen, wurde diese Verknüpfung explizit in den Mittelpunkt gestellt. Wurden im Sinne der Akteurs-Netzwerk-Theorie verschiedene Akteure auf eine sie betreffende Problemlage aufmerksam gemacht, so muss im nächsten Schritt ihr Interesse für eine Lösung geweckt werden. Erst dann kann es zu einer sogenannten Zuschreibung bzw. Inskription der Rollen oder im gegenteiligen Fall auch zur Ablehnung der Netzwerkbildung kommen (vgl. CALLON 2006a, S. 151ff).

Im Fall der Bananenproduktion von La Palma wurde der erste Schritt zur Bewusstwerdung der Problemlagen des konventionellen Anbaues durch einige wenige Bäuerinnen und Bauern, die bereits Bio-Bananen produzierten, jedoch keinen ausreichenden Absatz für ihre Ernte fanden, gesetzt. Sie wandten sich an die Genossenschaft „Volcán de San Juán“ und warben um deren Interesse an der Vermarktung nachhaltig produzierter Bananen. Die Kooperative richtete als erste Genossenschaft daraufhin eine Bioproduktlinie ein und wagte den Versuch auf dem spanischen Markt. Heute wird diese ökologische Vermarktungsalternative über zahlreiche weitere Kooperativen auf La Palma betrieben. Verkauft wird die Bio-Banane derzeit hauptsächlich auf dem spanischen Markt, aber auch in Deutschland und der Schweiz (Interviews mit Brito Brito; Rasi-Rüger).

Hinsichtlich der Motive, die der Entstehung bzw. Erweiterung des gegenwärtigen Netzwerks der Bio-Banane zugrunde liegen, stehen sich einerseits ökonomische Anreize und andererseits ideologische Überzeugungen gegenüber. Besonders deutlich treten diese teils unterschiedlichen Ausgangsinteressen bei den Produzenten hervor.

Nachdem man den ersten Vertretern des ökologischen Landbaus auf La Palma vor rund 30 Jahren noch wenig Verständnis entgegenbrachte, gelten sie heute auf diesem Ge- 


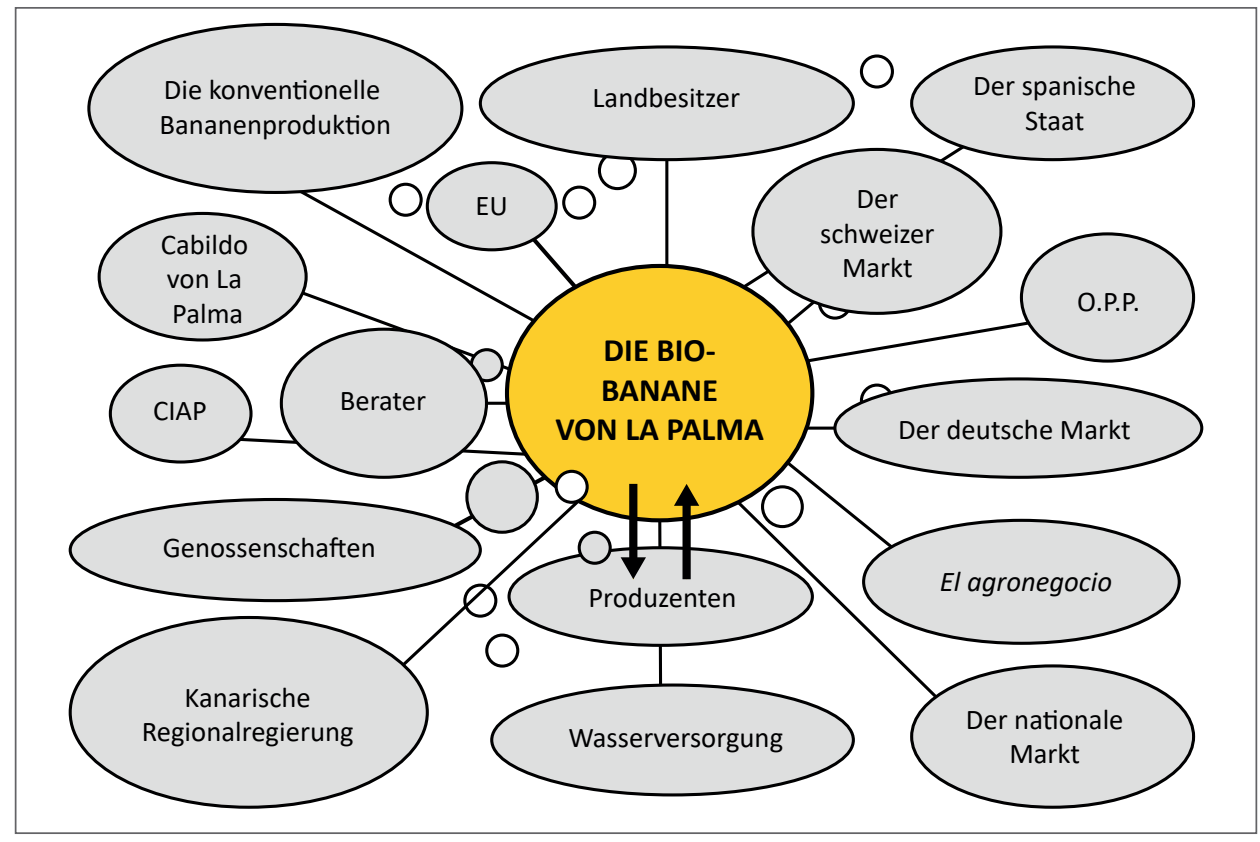

Quelle: Eigene Darstellung auf Basis von Unterlagen und Informationen von CIAP (Consejo Insular de Aguas de La Palma), O.P.P (Organizatión de Productores de Plátanos) und El Agronegocio (Anbieter von Dünge- und Pflanzenschutzmitteln)

Abb. 9: Die Hauptakteure des Akteurs-Netzwerks der Bio-Banane

biet als Pioniere. Ihrer Ansicht nach zielt der Bio-Anbau nicht nur darauf ab, die Umwelt zu schonen, sondern möchte auch die Unabhängigkeit der Produzenten steigern (Interview mit García Lázaro). Zudem bevorzugen manche Bananenbäuerinnen und -bauern grundsätzlich eine nachhaltigere Anbauweise, um nicht die eigene Gesundheit aufs Spiel zu setzen. Denn auf La Palma ist es nicht unüblich, dass sich die Finca in unmittelbarer Nähe des eigenen Wohnhauses befindet (Interview mit Méndez García).

Im Zuge dieser Entwicklungen spielte Ildefonso Acosta eine tragende Rolle, da er für interessierte Landwirte einen der ersten Kurse zum Bio-Bananenanbau anbot. Der Agraringenieur, der eine nachhaltige Düngemethode entwickelt hat, die vorrangig Ressourcen der eigenen Finca nutzt, kooperiert mit der Inselregierung, dem „Cabildo“ von La Palma, innerhalb des „Programa de Agricultura Sostenible“. Dieses Projekt, das um das Jahr 2007 ins Leben gerufen wurde, setzt sich die Unabhängigkeit der Landwirte sowie eine umweltschonende Landwirtschaft zum Ziel. Deshalb ist der Inselverwaltung nicht nur die Betreuung der Teilnehmer innerhalb des Netzwerks wichtig, sondern auch das Anwerben weiterer Produzenten. Diese Maßnahmen zielen auf eine langfristige Umstellung aus Überzeugung und nicht ausschließlich durch finanzielle Anreize oder im Sinne einer Steigerung der Produktivität ab. Dies impliziert auch die gezielte Vermittlung von Wissen, damit die Teilnehmer an Selbstständigkeit gewinnen können (Interview mit Batista Rodríguez). 
In den letzten Jahren konnte zudem beobachtet werden, dass sich vor allem junge Bäuerinnen und Bauern für die Bio-Landwirtschaft entscheiden, und zwar bewusst wegen des höheren Preises und der größeren Stabilität des Anbausystems. Daneben wurde auch eine „unbewusste Rückkehr“ zu einer wieder mehr nachhaltigen Landwirtschaft festgestellt. Denn manche Landwirte wollen ihre Ausgaben verringern und verzichten so auf den Einsatz von Handelsdüngern oder Pflanzenschutzmitteln. Mittlerweile betragen die Kosten für den Zukauf diverser Produkte nämlich bis zu 15 Prozent der Gesamtausgaben für einen durchschnittlichen konventionell geführten Betrieb (Interview mit Batista Rodríguez).

Aus der Abhängigkeit von den Subventionen sowohl für die ökologisch als auch für die konventionell produzierte Banane entstand eine Vernetzung der Produzenten, die sich „Para un Precio Justo del Plátano“ nennt. Die Initiatoren, zu denen sich auch Francisco García und Sara Hernández zählen, bezwecken mit dem Zusammenschluss von über 250 Bäuerinnen und Bauern eine Erhöhung der Flächenproduktivität. Jedoch ist bei manchen ökologisch wirtschaftenden Landwirten die Ansicht zu hören, dass Konzepte abseits der monetär gewinnbringenden Monokultur - also Pflanzungen in Mischkultur - nicht rentabel genug sein könnten.

„,Mittlerweile gibt es Leute, die wegen ökonomischer Gesichtspunkte einsteigen. “ (García Lázaro) $)^{7}$

„Es gibt eben auch diejenigen, die sich wegen dem höheren Preis für den biologischen Anbau entscheiden. Das gefällt mir weniger. " (Brito Brito) ${ }^{8)}$

Im Zuge der Interviews mit den Produzenten wurde auch von zukünftigen Plänen zum Ausbau der Produktion berichtet. Neben Bio-Landwirten, die ihre Finca gleichsam auf dem Status Quo beibehalten möchten, gibt es seitens der Interviewten unterschiedliche Ambitionen: Einige zielen auf eine Erhöhung der Flächenproduktivität ab, andere möchten die Anbaufläche erweitern. Obwohl die Steigerung der Produktion pro Finca auch ohne eine flächenmäßige Ausdehnung vonstatten gehen kann, befinden sich derzeit viele Produzenten auf der Suche nach einem weiteren geeigneten Grundstück für den Bio-Bananenanbau (Interview mit Pérez Díaz; Méndez García).

Der relativ geringe Bio-Anteil an der gesamten Bananen-Produktion auf La Palma lässt mutmaßen, dass sich der Großteil der so produzierenden Bäuerinnen und Bauern aus umweltethischen und nicht aus rein monetären Gründen zum Umstieg entscheidet. Viele von ihnen glauben an die Grundwerte der Bio-Landwirtschaft (Interview mit Rasi-Rüger). Trotzdem wird es wohl immer auch Akteure geben, die in der Umstellung auf Bio-Bananen-Produktion zentral die ökonomischen Vorteile sehen.

„,Die Mehrheit betreibt biologische Landwirtschaft aus philosophischer Überzeugung, denn insgesamt stellen die Bio-Bauern noch eine Minderheit dar. [...] Viele Menschen tun es aus Gewissen, nein aus Lebensphilosophie. [...] Und daneben

\footnotetext{
7) Spanischer Originaltext: „Y ahora hay gente ya que por cuestión económica empieza a meterse. “

8) Spanischer Originaltext: „, Y después hay otra gente, que me gusta menos eso. Que se metió en el cultivo ecológico por el tema del precio, sabes. “
} 
gibt es natürlich auch jene, die sich wegen der ökonomischen Vorteile dafür entschieden haben. " (Lorenzo Rodríguez) $)^{9)}$

Auch unter den Kooperativen lassen sich unterschiedliche Motive für die Vermarktung der Bio-Banane finden. Während AGUSA („Cooperativa Platanera de La Palma“) vorrangig auf die Steigerung der Rendite durch eine ökologische Linie abzielt, betont Rodrigo Brito von „Volcán de San Juán“ den großen Stellenwert der flächenmäßigen Ausdehnung der biologischen Landwirtschaft durch Überzeugung. Für beide Motive bestehen hohe Zukunftserwartungen - bis hin zu einer ökologischen Umstellung des Großteils der Bananenfincas. Betont wird von beiden befragten Geschäftsführern der Genossenschaften, dass eine Kooperative grundsätzlich als Unternehmen zu betrachten sei und daher wohl immer Interesse an Rendite und Kostendeckung bestehen wird (Interviews mit Arenciaba Sánchez; Brito Brito).

\section{„Auch den Kooperativen ist klar, dass die Zukunft im biologischen Anbau liegt.“ (Brito Brito) $)^{10)}$}

Innerhalb der O.P.P. COPLACA kümmern sich die Agraringenieure um die Steigerung der Anzahl der Bio-Bananenproduzenten, und zwar aus Überzeugung. Den Ingenieuren Javier López-Cepero und Manolo Puerta González sind daneben aber auch eine Verbesserung der Qualität des Anbaus durch innovative Produktionsmethoden wie auch der Kommunikation zwischen Landwirten und Genossenschaften wichtig. Außerdem wollen sie sich für eine verpackungsfreie Vermarktung der Bio-Banane einsetzen. Ihrer Meinung nach geht der Verbrauch zusätzlicher Ressourcen (z. B. Kunststoffeinpackungen der Fruchtstände) nicht konform mit den grundsätzlichen Zielen einer ökologischen Produktion. Somit lassen sich auch Bestrebungen seitens der O.P.P. finden, das Netzwerk der Bio-Banane von La Palma nicht nur größer werden zu lassen, sondern auch ökologisch und sozial nachhaltiger.

Da die Nachfrage nach biologischen Lebensmitteln europaweit ansteigt, will auch die Provinzregierung der Kanarischen Inseln („Gobierno de Canarias“) ihre Aufmerksamkeit auf diese Landwirtschaftsform richten. Sie forciert die Umstellung konventioneller Bananenpflanzungen auf Bio-Betriebe und zielt dabei auf eine schrittweise und langfristige Entwicklung ab. Dies inkludiert die primäre Umstellung auf eine integrierte Produktion, um in einem nächsten Schritt gänzlich nach den Kriterien des Biolandbaus zu produzieren. Welcher Motivation die Produzenten dabei nachgehen, spielt für die Regierung im Grunde genommen keine Rolle. Wünschenswert wäre zwar eine Umstellung aus Überzeugung, erfolgt diese jedoch rein aus finanziellen Motiven, so betrachtet die Regierung dies auch als einen legitimen Grund (Interview mit Martín Pérez).

Darüber hinaus entdecken auch die Anbieter der Dünge- und Pflanzenschutzmittel, also das Netzwerk des sogenannten „agronegocio“, einen Vorteil in der Expansion der

\footnotetext{
9) Spanischer Originaltext: „Hay más de filosofia porque la agricultura ecológica todavía es minoritaria. [...] mucha gente lo hace por conciencia, no, por filosofia de vida. [...] Y después, pues hay gente también que se ha ido por cuestión económica, vale."

${ }^{10)}$ Spanischer Originaltext: „Pero si desde dentro, desde las cooperativas, tienen claro, lo que yo te acabo de decir, que el futuro va por el tema ecológico. “
} 


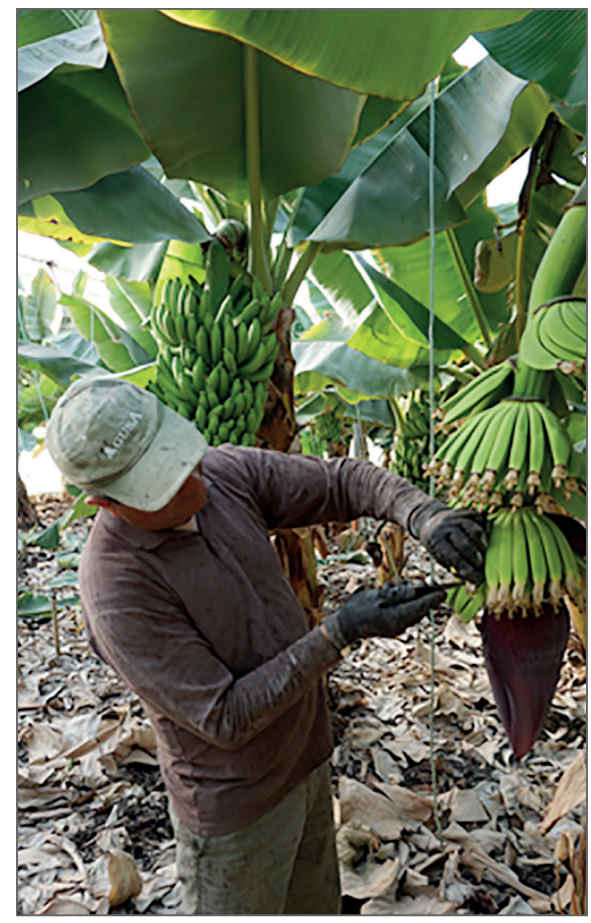

Quelle: Foto: WIPLINGER 2019

Abb. 10: Arbeit auf der Bananenparzelle - Entfernen der Blütenknospen jedes einzelnen Bananenfingers während der Reifung der Fruchtbüschel

Bio-Banane auf La Palma. Denn so wie es ein großes Angebot an diversen Produkten für die konventionelle Landwirtschaft gibt, so beginnt auch das Sortiment im Biobereich zu wachsen. Die Anbieter passen ihre Produktlinie an die steigende Tendenz zur biologischen Landwirtschaft an. Während im konventionellen Anbau einfach auf chemisch-synthetische Mittel zur Bearbeitung der Pflanzung zurückgegriffen wird, können die Landwirte nach erfolgtem Umstieg zur biologischen Landwirtschaft einen erforderlichen Düngebzw. Pestizideinsatz durch eine ökologische Alternative ersetzen. Der Geschäftsführer von „Volcán de San Juán“ spricht mittlerweile sogar davon, dass die Mehrheit der Produkte in seiner Kooperative für den ökologischen Anbau zertifiziert ist. Inbegriffen sind dabei zum Verkauf angebotene Pflanzenschutz- und Düngemittel.

„, Wenn du heutzutage in das Lager unserer Genossenschaft kommst, wirst du sehen, dass fast alle Produkte biologisch sind. Sogar die Düngemittel werden umgestellt. "(Brito Brito) $)^{11)}$

\footnotetext{
11) Spanischer Originaltext: „Ahora ya entras [Anm.: en el almacén] y casi todos los productos son ecológicos. [...] y los abonos se están convirtiendo en ecológico también. “
} 
Die Anbieter orientieren sich nicht nur am Trend zur biologischen Landwirtschaft, sondern zielen auch auf eine allgemeine Erhöhung der Gewinnspanne ab, da sie für die ökologischen Produkte höhere Beträge verlangen können.

„, Sie erkennen schön langsam die ökonomischen Möglichkeiten des biologischen Marktes, da er sogar rentabler als der konventionelle ist. Die Gewinnspanne ist hier höher, weil die Produkte teurer verkauft werden können. " (García Lázaro) ${ }^{12)}$

In jüngerer Zeit kann auch ein reges Interesse des Schweizer Markts an dem Akteur-Netzwerk beobachtet werden. Derzeit erfolgen im Rahmen eines Pilotprojekts bereits regelmäßig Lieferungen. Die Konsumenten in der Schweiz nehmen die Bio-Banane von La Palma sehr gut an, und die Nachfrage steigt. Dementsprechend möchten die Händler die Liefermenge kontinuierlich steigern. Zudem zeigen auch Detaillisten einer Supermarktkette in Deutschland Interesse an der Einführung der Bio-Banane von La Palma (Interview mit Arenciaba Sánchez).

In indirekter Weise mit der Bananenproduktion von La Palma verbunden ist auch der „Consejo Insular de Aguas de La Palma“ (CIAP, Inselrat für die Wasserversorgung). Dessen Präsident José Perestelo ist sich über den wirtschaftlichen Stellenwert der Bananenexporte wie auch der Rolle des Wassers für die Kultivierung bewusst. Somit setzt sich der CIAP zum Ziel, jetzt wie auch in Zukunft die Wasserbestände für agrarische Zwecke zu kontrollieren, zu schützen und die nötige Infrastruktur dafür instandzuhalten. Mithilfe von Investitionen sollen Wasserverlusten vorgebeugt und neue Gewinnungsarten wie die Abwasseraufbereitung eingeführt werden. Zudem soll eine ausgewogene und faire Verteilung der Wasserressourcen in allen Sektoren erreicht werden (Interview mit Martín Pérez).

Als Akteure, die kein oder nur ein mäßiges Interesse an der Ausbreitung des Netzwerks der Bio-Banane auf La Palma haben, lassen sich die konventionelle Bananenproduktion und der nationale Markt nennen. Da vor allem die Nachfrage des spanischen Marktes nach der Bio-Banane stagniert, stellen sich die Märkte anderer europäischer Staaten als wichtige Zielmärkte für die Bio-Genossenschaften heraus. Hinter der konventionellen Banane verbirgt sich eine starke Lobby, die eine weitere Ausbreitung des Netzwerks nicht im Einklang mit den eigenen wirtschaftlichen Plänen sieht und eine Verschlechterung der Ressourcensituation bereitwillig in Kauf nimmt. Unter dementsprechender Beeinflussung durch diese Gruppe lehnen viele der konventionellen Bäuerinnen und Bauern den Ökolandbau nach wie vor grundsätzlich ab oder zeigen sich ihm gegenüber zumindest sehr skeptisch (Interview mit Rasi-Rüger).

\section{Diskussion und Fazit}

Im Zuge der Tiefeninterviews mit Persönlichkeiten aus den Bereichen Produktion, Vermarktung und Administration wurde - flankierend zu explorativen Beobachtungen - er-

\footnotetext{
${ }^{12)}$ Spanischer Originaltext: „Ellos están empezando a ver el negocio del ecológico porque hasta es más beneficioso. Da más renta que lo otro, es más caro. “
} 
hoben, inwiefern die beteiligten Akteure die Entwicklung der Bio-Banane fördern und welche Konflikte und Kontroversen dem bestehenden Akteurs-Netzwerk in welcher Weise Grenzen setzen. Der Ansatz der Untersuchung und deren Ergebnisauswertung wurden in die Akteur-Netzwerk-Theorie eingebettet.

Die Untersuchung ergab, dass sich die existenziellen Rahmenbedingungen des Netzwerks der Bio-Banane sowohl in der Ressourcenfrage als auch in den wechselseitigen Verknüpfungen zahlreicher Akteure manifestieren. Die Ressourcen gliedern sich in materielle Mittel, zu denen vorrangig Wasser, Boden, Land, Nährstoffe und Pflanzenschutzmittel innerhalb der Produktion gehören. Dabei erfolgen diese Inputs durch das Netzwerk der Wasserversorger, Landbesitzer bzw. der jeweiligen Finca sowie den kommerziellen Handel mit Dünge- und Pflanzenschutzmitteln. Bezüglich der Vermarktung durch die Genossenschaften stellen Wasser, Energie, Pflanzenschutzmittel, Plastik und Karton die zentralen Ressourcen dar.

$\mathrm{Zu}$ den immateriellen Mitteln gehört neben dem Arbeitseinsatz das Know-how rund um die Methoden der Bio-Landwirtschaft. Generiert wird dieses entweder durch die Produzenten selbst, durch Fortbildungsmaßnahmen (z. B. durch Agraringenieur Ildefonso Acosta), durch den „Cabildo“ von La Palma, durch Genossenschaften oder den O.P.P., die Organisation der Produzenten. Weitere immaterielle Rahmenbedingungen sind das existierende Nachfrageinteresse von Seiten des spanischen, Schweizer und deutschen Marktes, die rechtlichen Verordnungen der EU wie auch die spanische Gesetzgebung. Ebenso tragen die jeweiligen Vorschriften der Genossenschaften sowie die Politik der Kanarischen Regionalregierung und des lokalen Wasseramtes (CIAP) zum Bestehen bzw. Funktionieren des Netzwerks bei.

Die Summe von Akteuren und Ressourcen bildet auf Basis dieser Rahmenbedingungen das Akteur-Netzwerk der Bio-Banane von La Palma. Trotz des Bestehens eines relativ stabilen Netzwerks treten divergierende Absichten in Erscheinung. So stehen sich vor allem jene Akteure gegenüber, die sich aus umweltethischer Überzeugung für den ökologischen Anbau einsetzen, und solche, die sich vorrangig ökonomische Vorteile davon versprechen und ihre Bio-Bananenpflanzungen monokulturell betreiben.

Insgesamt zeigen sich bei tiefgehender Analyse des Akteur-Netzwerkes der Bio-Banane von La Palma diverse Ungleichheiten. Diese manifestieren sich vor allem in der zumeist machtlosen Position der Produzenten. So müssen sie mangels Alternativen die von den Genossenschaften initiierten Handlungspraktiken akzeptieren. Als Konsequenz entstehen den Landwirten vor allem Einnahmenverluste. Auch hinsichtlich der Wertschöpfung zeigt sich, dass die Produzenten im Vergleich zu den Zwischenhändlern den geringeren Anteil am Erlös erhalten. Mögliche Lösungen wie etwa der Direktverkauf werden vor allem durch die geringe Nachfrage des lokalen Marktes begrenzt. Auch die fehlende Vernetzung der Bio-Produzenten untereinander bzw. das dissidente Verhalten mancher von ihnen schwächen ihre Verhandlungsposition.

Der agrochemischen Industrie gelingt es, weiterhin einige der Bio-Bäuerinnen und Bio-Bauern an sich zu binden. Anklang finden vor allem organische Fertigdünger einerseits aus Gewohnheit, andererseits durch das fehlende Wissen über Alternativen. Letzteres versuchen wiederum die Agraringenieure des Cabildo von La Palma mithilfe von Fortbildungsmaßnahmen wettzumachen. Durch zusätzliches Know-how können die 
Landwirte von externen Inputs, die ihr Budget unnötig belasten, unabhängiger werden. Allerdings sind die Agrochemie-Firmen mit diesen Fortbildungsmaßnahmen nicht einverstanden, wodurch eine konfliktreiche Beziehung zur Inselregierung entstanden ist. Eine weitere Abhängigkeit der Produzenten besteht hinsichtlich der Wasserversorgung der Insel. Die Interviews brachten hervor, dass durch ein nachlässiges Management des „Consejo Insular de Aguas de La Palma“ die Stabilität der Wasserversorgung wie auch deren Qualität nicht durchgängig gewährleistet werden kann, was insbesondere in Trockenperioden ein Problem für die Bananenproduzenten darstellt.

Im Bereich der Vermarktung kann die Bio-Banane vom hohen Organisationsgrad und der stabilen Verbindung zwischen den einzelnen Genossenschaften profitieren. Doch auch deren Möglichkeiten werden Grenzen gesetzt. Als machtlos zeigen sie sich vor allem gegenüber der EU, die durch Verordnungen zahlreiche obligatorische Richtlinien schuf, die allerdings auch zu einer sehr stabilen „Repräsentation“ innerhalb des Akteur-Netzwerks der Bio-Banane führten. Daneben festigte sich eine weitere Machtasymmetrie zwischen Genossenschaften, Produzenten und dem nationalen Markt, da dieser mithilfe einer Quote die gehandelten Mengen wie auch den Preis der Bananen bestimmt.

Das Zustandekommen weiterer zukunftsfähiger Netzwerkstrukturen hängt zudem von den konventionellen Produzenten ab. Entscheiden sich mehr Landwirte für einen biologischen Anbau, so steigt die Produktionsmenge und erleichtert dadurch die Erschließung weiterer internationaler Märkte. Dafür förderlich kann die zunehmende Sensibilisierung der jüngeren Generation der Landwirte für ökologische Themen sein. Es stehen bereits zur Genüge Erfahrungswerte und „Good Practice“-Beispiele zur Verfügung, die auch gegen Unwissen bzw. falsche Vorstellungen gegenüber der Bio-Landwirtschaft eingesetzt werden können. Zudem kann die EU durch weitere obligatorische Richtlinien-Einschränkungen der möglichen Pflanzenschutzmittel die Bio-Landwirtschaft als potenzielle Alternative attraktiver erscheinen lassen.

Darüber hinaus zeichnet sich auf La Palma eine Konkurrenzsituation der ökologischen zur integrierten Produktion ab. Sowohl die Kanarische Regionalregierung als auch die Genossenschaften zielen vorrangig auf eine schrittweise Abkehr von der konventionellen Produktion ab, indem sie aktiv die integrierte Landwirtschaft fördern, um die Basisressourcen für die Bananenwirtschaft zu schonen. Ändert sich im Zusammenhang mit der Entwicklung der sich wandelnden Niederschlagsverteilung in Zeiten des Klimawandels an der aktuellen Situation nichts, so werden künftig auch Überlegungen zu wassersparenderen Landwirtschaftskonzepten, wie es etwa die Permakultur sein kann, unausweichlich. Weitere mögliche Hindernisse für das Funktionieren des Netzwerkes zeigen sich in der Abhängigkeit der Produzenten von den Subventionen. Zudem besteht trotz der aktuellen Preisstabilität das Risiko, dass der Marktwert der Bio-Banane nach dem Vorbild der konventionellen Produktion sinkt bzw. an Instabilität zunimmt.

Damit zukünftige Stärken genutzt und mögliche Hindernisse erfolgreich aus dem Weg geräumt werden können, bedarf es einer Harmonisierung der Interessen aller Beteiligten. Um eine gemeinsame Zielvorstellung langfristig umsetzen zu können, müssen zunächst die Motivationen der relevanten Akteure in Einklang gebracht und folglich ihre Rollen klarer definiert werden. Können sich die Akteure auf ein gemeinsames Handlungsprogramm einigen, so ist eine noch stabilere Entwicklung für alle Beteiligten 
möglich. Es ist vor allem eine intensivere Vernetzung der Produzenten von Bedeutung, da sie innerhalb des Netzwerks die schwächste Stellung innehaben. Durch mehr und stärkere Verknüpfungen können sie zudem Synergien nutzen und Ressourcen einsparen. Vor allem im Bereich der Dünge- und Pflanzenschutzmittel besteht noch großes Einsparpotenzial.

Insgesamt können ein professionell moderierter Abbau der Machtasymmetrien und eine Begegnung der Beteiligten auf Augenhöhe maßgeblich zu einer sozial, ökologisch und ökonomisch ausbalancierten Entwicklung des Bio-Bananenanbaus auf La Palma sowie des damit verbundenen politischen Umfeldes beitragen. Beispielsweise könnten Verantwortliche des Biosphärenparks diesen Prozess proaktiv moderieren oder zumindest begleiten. In Summe stellt die Bio-Banane von La Palma im Sinne der Akteur-Netzwerk-Theorie eine stabile „Repräsentation“ dar. Durch das Eingehen zusätzlicher Verbindungen - z. B. „Interessement“ des Schweizer Marktes, stärkere Einbindung der Biosphärenparkpolitik - würde es möglich sein, die Stabilität des Netzwerkes zukünftig noch zu erhöhen. Wie dies konkret in der Bananenwirtschaft auf La Palma gelingen könnte, dazu wären über die vorliegende Arbeit hinausführende vertiefende Untersuchungen der Governance-Strukturen erforderlich.

\section{Literaturverzeichnis und Anhang}

Acosta A. (1999): Canarias contará en los próximos años con 4.500 hectáreas para la agricultura ecológica [Die Kanaren werden in den kommenden Jahren mit 4.500 Hektar für die biologische Landwirtschaft rechnen können]. In: Revista de Medio Ambiente [Umweltmagazin], 14, S. 23-25.

AEMET - Atlas Climático de los Archipiélagos de Canarias, Madeira y Azores [Klimatischer Atlas des Archipels der Kanarischen Inseln, Madeiras und der Azoren] (2012). Madrid: Agencia Estatal de Meteorologia (AEMET). - http://www.aemet.es/es/conocermas/recursos_en_ linea/publicaciones_y_estudios/publicaciones/detalles/segundo_Atlas_climatologic ( $\mathrm{Zu}-$ griff: 11.02.2019).

ASPROCAN - Asociación de Organizaciones de Productores de Plátanos de Canarias [Verband der Organisationen der Bananenproduzenten der Kanarischen Inseln] (o. J.). - http://igpplatano decanarias.es/ (Zugriff: 28.02.2019).

ASPROCAN - Asociación de Organizaciones de Productores de Plátanos de Canarias) [Verband der Organisationen der Bananenproduzenten der Kanarischen Inseln] (2018): Dossier Exportation Plátanos de Canarias [Exportkatalog Kanarische Banane]. - https://platanodecanarias. es/wp-content/uploads/2018/11/PdC_FA18_DossierExportacion_170x240mm_ale_web.pdf (Zugriff: 14.05.2019).

Berners-Lee M. (2010): How Bad Are Bananas? The Carbon Footprint of Everything. London: Profile Books Ltd.

Benjamin D., Virkler L. (2016): Farm to Table. The Essential Guide to Sustainable Food Systems for Students, Professionals, and Consumers. White River Junction, VT: Chelsea Green Publishing.

BOE - Boletín Oficial del Estado (1990): Ley 12/1990, de 26 de julio, de Aguas [Gesetz 12/1990 vom 26. Juli über die Wasserversorgung]; - auch online unter: https://www.boe.es/buscar/ pdf/1990/BOE-A-1990-23087-consolidado.pdf (Zugriff: 20.02.2019). 
Callon M. (2006a): Einige Elemente einer Soziologie der Übersetzung: Die Domestikation der Kammmuscheln und der Fischer der St. Brieuc-Bucht. In: Belliger A., Krieger D. J. (Hrsg.): ANThology. Ein einführendes Handbuch zur Akteur-Netzwerk-Theorie. Bielefeld: Transcript, S. 135-174.

Callon M. (2006b): Techno-ökonomische Netzwerke und Irreversibilität. In: Belliger A., KrIEGER D. J. (Hrsg.): ANThology. Ein einführendes Handbuch zur Akteur-Netzwerk-Theorie. Bielefeld: Transcript, S. 309-342.

Cook I. (2004): Follow the Thing: Papaya. In: Antipode, 36, S. 642-664.

Colombino A. (2014): The Geography of Food. In: Bollettino della Società Geografica Italiana Serie 12,7 (4), S. 647-656.

CIAB - Fundación Canaria Centro Internacional de Agricultura Biológica (2019): Fundación CIAB: Un impulso para la agricultura ecológica en La Palma [Stiftung CIAB: Ein Impuls für die biologische Landwirtschaft auf La Palma]. La Palma: CIAB. - http://www.fundacionciab. com/impulso-agricultura-ecologica/ (Zugriff: 09.05.2019).

CIAP - Consejo Insular de Aguas de La Palma (Hrsg.) (2017): Plan hidrológico de La Palma. Ciclo de Planificación Hidrológica 2015-2020 [Hydrologischer Plan von La Palma. Zeitraum der Hydrologischen Planung 2015-2020]; - auch online unter: http://www.gobiernodecanarias.org/ politicaterritorial/descargas/PHIL_2_Ciclo_La_Palma/ES125_PROY_PLAN_La_Palma_ ANEJO1_VB.pdf (Zugriff: 20.02.2019).

Dinerstein E., Olson D., Joshi A., Vynne C, Burgess N. D. et al. (2017): An Ecoregion-Based Approach to Protecting Half the Terrestrial Realm. In: BioScience, 67 (6), S. 534-545.

Dodo M. K. (2009): Evolución del sector del plátano en Canarias tras la creación y reformas de la OCM [Entwicklung des Bananensektors der Kanaren nach der Gründung und Reformierung der OCM]. In: Boletín Económico de ICE, Información Comercial Española, 2973, S. 17-26.

Drehsing T., Pehl T. (2018): Praxisbuch Interview, Transkription \& Analyse. Anleitungen und Regelsysteme für qualitativ Forschende. 8. Aufl., Marburg: dr. dresing \& pehl GmbH.

Europäische Kommission (Hrsg.) (2010): Mitteilung der Kommission an den Rat und das europäische Parlament. Begleitmaßnahmen für den Bananensektor: Unterstützung der nachhaltigen Anpassung der wichtigsten AKP-Bananenexportländer an das neue Handelsumfeld. - http://ec.eu ropa.eu/transparency/regdoc/rep/1/2010/DE/1-2010-101-DE-F2-1.Pdf(Zugriff: 14.02.2019).

Europäische Kommission (Hrsg.) (2019): Banana Supply in the EU. - https://ec.europa.eu/agri culture/sites/agriculture/files/fruit-and-vegetables/product-reports/bananas/statistics/supply_ en.pdf (Zugriff: 14.05.2019).

Europäische Kommission (Hrsg.) (o. J.): Quality Schemes Explained. Aims of EU Quality Schemes. https:/lec.europa.eu/info/food-farming-fisheries/food-safety-and-quality/certification/qualitylabels/quality-schemes-explained_en\#logos (Zugriff: 28.02.2019).

Europäisches Parlament und Rat der Europäischen Union (Hrsg.) (2012): Amtsblatt der Europäischen Union, L 343, 14. Dezember 2012. Brüssel: Europäische Union.

Gobierno de Canarias [Regierung der Kanarischen Inseln] (Hrsg.) (2014): Plan de Regadíos de Canarias [Bewässerungsplan der Kanaren]. - http://www.gobiernodecanarias.org/agricultura/ docs/desarrollo-rural/regadio/6_PRC_La_Palma.pdf (Zugriff: 03.05.2019).

Gobierno de Canarias [Regierung der Kanarischen Inseln] (Hrsg.) (2015): Programa Comunitario de Apoyo a las producciones agrarias de Canarias [Gemeinschaftsprogramm zur Unterstützung der landwirtschaftlichen Produktion der Kanaren]. - http://www.gobiernodecanarias.org/ agricultura/doc/otros/posei/POSEI_Canarias-Programa_Agrario-Consolidado_enero_ 2015.pdf (Zugriff: 14.05.2019).

Gobierno de Canarias [Regierung der Kanarischen Inseln] (Hrsg.) (2016): Agricultura Ecológica. Resumen de los principales Datos 31/12/2016 [Biologische Landwirtschaft. Zusammenfas- 
sung der wichtigsten Daten 31/12/2016]. - http://www.gobiernodecanarias.org/cmsgobcan/ export/sites/agricultura/icca/galerias/doc/calidad/DATOS_AGRICULTURA_ECOLOGICA_ 2010_2016.pdf (Zugriff: 21.02.2019).

Gobierno de Canarias [Regierung der Kanarischen Inseln] (Hrsg.) (2018): Informe estadístico. Mapa de cultivos de La Palma (2017) [Statistische Mitteilung. Karte der Anbausorten von La Palma (2017)]. - auch online unter: http://www.gobiernodecanarias.org/agricultura/doc/ desarrollo-rural/doc/LP/camp-2017/informe_lp_2017.pdf(21.02.2019).

Gobierno de Canarias [Regierung der Kanarischen Inseln] (Hrsg.) (2019): Agricultura ecológica [Biologische Landwirtschaft]. - http://www.gobiernodecanarias.org/agricultura/icca/temas_calidad/agricultura_ecologica/ (Zugriff: 21.02.2019).

Hammer T., Mose I., Siegrist D., Weixlbaumer N. (2018): Großschutzgebiete in Europa im Wandel - Herausforderungen und Perspektiven für die Schutzgebietsentwicklung. In: Natur und Landschaft, 93 (5), S. 224-231

Hernández Luis J., González Morales A., Hernández Torres S., Ramón Ojeda A. (2017): El impacto del turismo de masas en las Islas Canarias en el contexto de las reservas mundiales de la biosfera [Der Einfluss des Massentourismus auf die Kanarischen Inseln im Kontext der Biosphärenreservate]. In: Cuadernos de Turismo [Tourismus Notizen], 40, S. 363-387.

ISTAC - Instituto Canario de Estadística [Institut für Statistik der Kanarischen Inseln] (Hrsg.) (2019): Estadísticas por temas [Statistiken nach Themen]. - http://www.gobiernodecanarias. org/istac/temas_estadisticos/(Zugriff: 19.06.2019).

Koeppel D. (2008): Banana. The Fate of the Fruit that Changed the World. London: Penguin.

Latour B. (2010): Eine neue Soziologie für eine neue Gesellschaft. Einführung in die AkteurNetzwerk-Theorie. Frankfurt am Main: Suhrkamp.

LóPEZ-CEPERo JimÉNeZ J. (2006): ¿Plátano ecológico? [Biologische Banane?] - In: Rincones del Atlántico [Atlantische Gegenden], 2006/3, S. 266-271.

López-Cepero Jiménez J., Puerta M., Rodríguez-Romero A. S. (2006): Factores limitantes de la producción de platano ecológico en las Islas Canarias [Limitierende Faktoren im biologischen Bananenanbau der Kanarischen Inseln]. - https://www.agroecologia.net/recursos/ publicaciones/publicaciones-online/2006/CD\%20Congreso\%20Zaragoza/Ponencias/ 78\%20LopezCepero\%20Com-\%20Factores.pdf (Zugriff: 20.05.2019).

Marzol JAÉn V., MÁyer SuÁrez P. (2012): Algunas reflexiones acerca del clima de las Islas Canarias [Einige Reflexionen in Bezug auf das Klima der Kanarischen Inseln]. In: Nimbus: Revista de climatología, meteorología y paisaje [Nimbus: Zeitschrift für Klimatologie, Meteorologie und Landschaft], 29-30, S. 399-416.

MAYrIng P. (2010): Qualitative Inhaltsanalyse. Grundlagen und Techniken. 11., aktualisierte und überarbeitete Ausgabe. Weinheim / Basel: Beltz.

Ministerio de Agricultura, Pesca y Alimentación (Hrsg.) (2012): Agricultura, Alimentación y Medio Ambiente en España 2011 [Landwirtschaft, Ernährung und Umwelt in Spanien 2012]. Madrid: Goberno de España.

Ministerio de Agricultura, Pesca y Alimentación (Hrsg.) (2019): Informa Anual de Indicatores: Agricultura, Pesca y Alimentación, Ano 2018 [Jahresbericht der Indikatoren: Landwirtschaft, Fischerei und Ernährung, Jahr 2018]. Madrid: Goberno de España.

Peuker B. (2011): Akteur-Netzwerk-Theorie und Politische Ökologie. In: Gross M. (Hrsg.): Handbuch Umweltsoziologie. Wiesbaden: Springer, S. 71-91.

Piatti Farnell L. (2016): Banana - A Global History. Chicago: University of Chicago Press.

Robinson J. C., Galán Saúco V. (2011): Plátanos y Bananas [Kanarische Bananen und Bananen]. Madrid: Mundiprensa. 
Rodríguez-Martín J., Santamarta J. C. (2013): Introduction to Water Problems in Canary Islands. In: Santamarta J. C., Gutiérrez L. E. (Hrsg.): Environmental Security, Geological Hazards and Management. San Cristóbal, Teneriffa: Universidad de La Laguna, S. 169-179.

Rodríguez Brito W. (2016): Conflictos por el uso del agua en Canarias [Konflikte rund um die Wassernutzung auf den Kanaren]. In: Universidad de Alicante (Hrsg.): Libro jubilar en homenaje al profesor Antonio Gil Olcina. Edición Ampliada [Festschrift zur Hommage an Professor Antonio Gil Olcina]. Sant Vicent del Raspeig: Universidad de Alicante, S. 249-266.

Rodríguez GonzÁlez P., Hernández-Martín R., Rodríguez Rodríguez Y., Baute Díaz Nisamar (2016): Los pilares de la Economía de La Palma [Die Eckpfeiler der Wirtschaft von La Palma]. - https://www.asdetur.com/files/pilares-economia-la-palma-def.pdf(Zugriff: 12.02. 2019).

Rотне P. (2008): Kanarische Inseln. Lanzarote, Fuerteventura, Gran Canaria, Tenerife, Gomera, La Palma, Hierro. Berlin / Stuttgart: Gebr. Bornträger (= Sammlung geologischer Führer, 81).

Vотн A. (1997): Agrargeographie des tropisch-subtropischen Obstanbaus an der südlichen Peripherie der Europäischen Union. Dissertation, Universität Göttingen (= Sozialökonomische Schriften zur ruralen Entwicklung, Kiel, 118).

WeIXLBAUMER N. (2005): „Naturparke“ - Sensible Instrumente nachhaltiger Landschaftsentwicklung. Eine Gegenüberstellung der Gebietsschutzpolitik Österreichs und Kanadas. In: Mitteilungen der Österreichischen Geographischen Gesellschaft, 147, S. 67-100.

Wiplinger A. (2019): Die Banane im Einklang mit Mensch und Umwelt? Eine empirische Analyse des ökologischen Bananenanbaus auf La Palma im Spiegel der Akteur-Netzwerk-Theorie. Diplomarbeit, Universität Wien.

\section{Anhang}

Interviewpartner auf La Palma aus Produktion, Vermarktung und Administration (2019)

\section{Produktion:}

- Martina Rasi-Rüger (Produzentin)

- Francisco García Lázaro (Produzent)

- Daniel Pérez Díaz (Produzent)

- Sara Hernández Nuñez (Produzentin)

- Marta Méndez García (Produzentin)

\section{Vermarktung:}

- Carmelo Arencibia Sánchez (Geschäftsführer Genossenschaft AGUSA)

- Rodrigo Brito Brito (Geschäftsführer Genossenschaft Volcán de San Juán)

- Laura Brito Pérez (Stellvertr. Geschäftsführerin Genossenschaft Volcán de San Juán)

- Manolo Puerta González (Agraringenieur O.P.P. COPLACA)

- Javier López-Cepero Jiménez (Agraringenieur O.P.P. COPLACA)

\section{Administration:}

- Andrés Batista Rodríguez (Agraringenieur Cabildo La Palma)

- César Martín Pérez (Generaldirektor des Landwirtschaftsministeriums der Kanarischen Regionalregierung)

- Ricardo Lorenzo Rodríguez (Agraringenieur des Cabildo La Palma und Professor an der Landwirtschaftlichen Fachschule La Palma)

- Victor Gómez Rodríguez (Agraringenieur des Cabildo La Palma) 